\title{
Optical Fiber Sensor Instrumentation for Slagging Coal Gasifiers
}

\section{Final Report}

Reporting Period Start: 20 July 2005

Reporting Period End: 18 July 2008

Principal Investigators: Anbo Wang and Kristie Cooper

Date of Issue: $\quad$ October 2008

DOE Award Number: $\quad$ DE-FG26-05NT42532

Submitted by: $\quad$ Center for Photonics Technology

Department of Electrical and Computer Engineering Virginia Polytechnic Institute and State University 460 Turner Street NW, Suite 303

Blacksburg, VA 24061

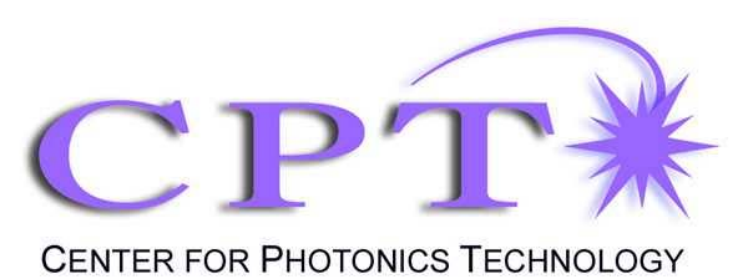




\section{Disclaimer}

This report was prepared as an account of work sponsored by an agency of the United States Government. Neither the United States Government nor any agency thereof, nor any of their employees, makes any warranty, express or implied, or assumes any legal liability or responsibility for the accuracy, completeness, or usefulness of any information, apparatus, product, or process disclosed, or represents that its use would not infringe privately owned rights. Reference herein to any specific commercial product, process, or service by trade name, trademark, manufacturer, or otherwise does not necessarily constitute or imply its endorsement, recommendation, or favoring by the United States Government or any agency thereof. The views and opinions of authors expressed herein do not necessarily state or reflect those of the United States Government or any agency thereof. 


\begin{abstract}
Coal gasifier is one of the most promising solutions for clean fossil energy. Refractory thickness monitoring and online real-time temperature measurement is needed for improved reliability and advanced process control for current and future generation power plants. The objective of this program is to design and implement an optical fiber based sensing system that could potentially be used to monitor refractory wall thickness and temperature inside a coal gasifier.

For the thickness monitoring, the system should be able to operate at temperatures up to $1000^{\circ} \mathrm{C}$. For this temperature range, silica fiber can still work so it is chosen for the sensor design. The measurement is based on a photon counting optical time domain reflectometer. A narrow light pulse is launched into a silica fiber which could be embedded into the gasifier refractory wall, and is partially reflected by the far end of the fiber. The time of flight of the light pulse in the fiber then gives an indication of the position of the fiber end, which is a function of the wall thickness when the fiber is embedded. Results obtained show a measurement accuracy of $\pm 2 \mathrm{~cm}$ in environment of $1000^{\circ} \mathrm{C}$ with a saw cut fiber end. When the fiber end is corroded by sodium carbide at $900{ }^{\circ} \mathrm{C}$, the accuracy is $\pm 3 \mathrm{~cm}$.

For the temperature measurement, a single crystal sapphire fiber sensor is designed. The sapphire fiber guides the broadband light from a light emitting diode to a sapphire wafer functioning as a Fabry-Perot interferometer and the wafer optical thickness is a function of temperature. The returned optical signal is then demodulated by multimode fiber based whitelight interferometry. The system was tested up to $1500^{\circ} \mathrm{C}$ with a measurement accuracy of $\pm 10^{\circ} \mathrm{C}$ for the entire measurement range.
\end{abstract}




\section{Table of Contents}

Abstract

Table of Contents

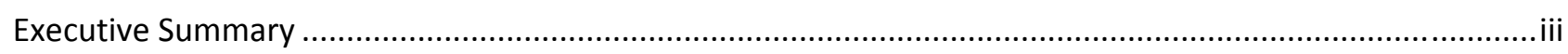

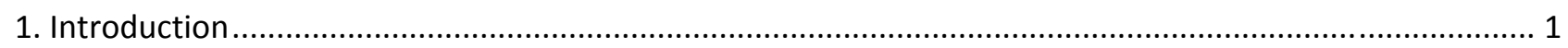

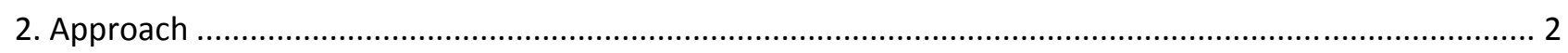

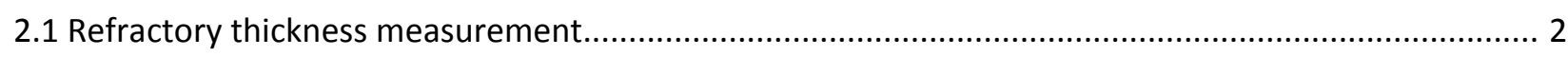

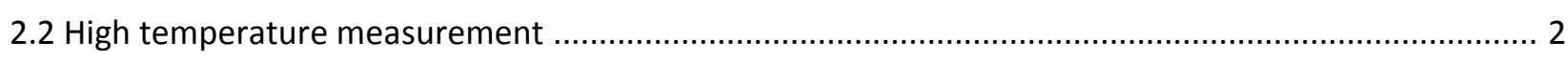

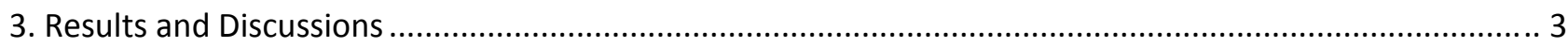

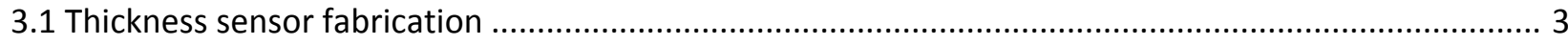

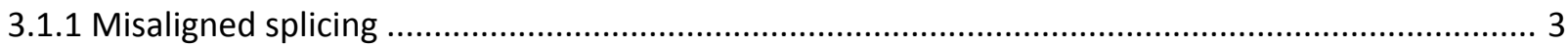

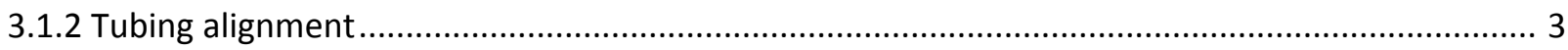

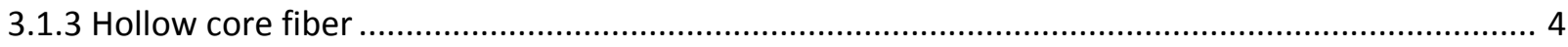

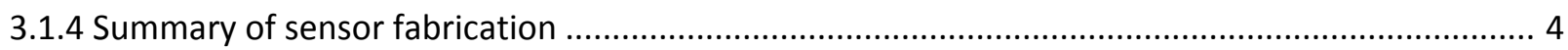

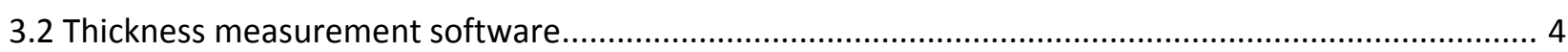

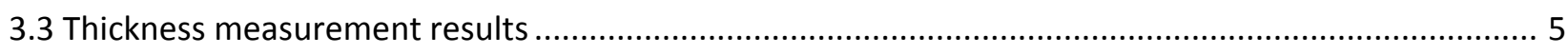

3.3.1 Fiber length measurement at room temperature.................................................................... 5

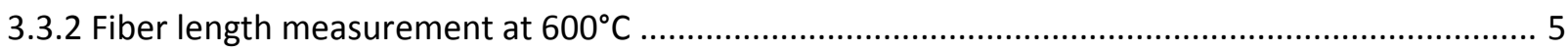

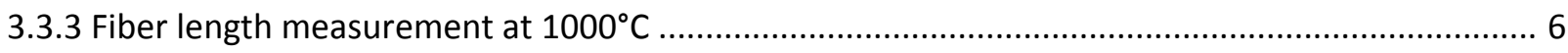

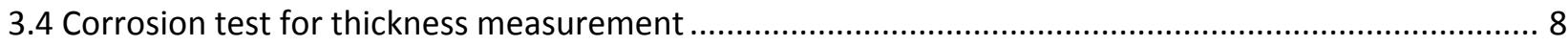

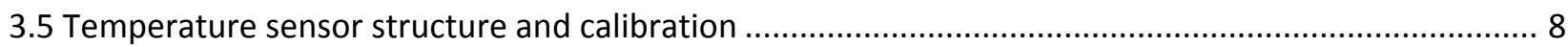

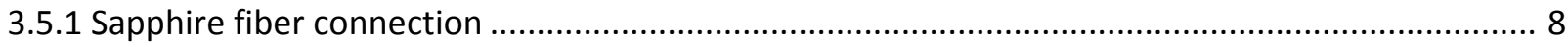

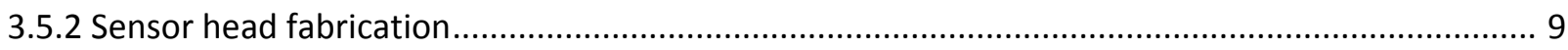

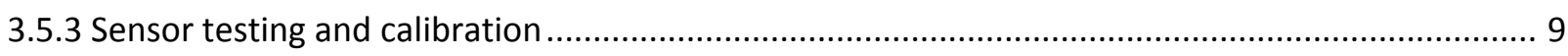

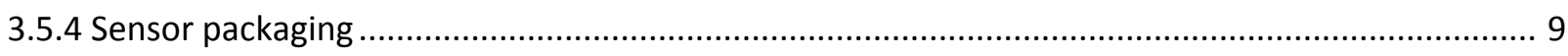

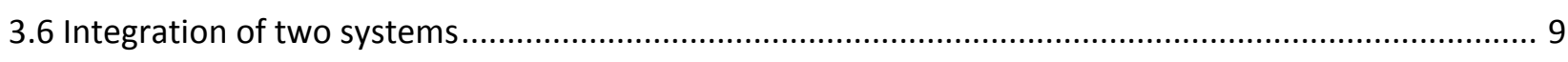

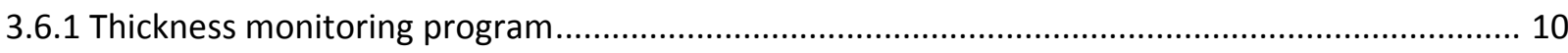

3.6.2 Temperature measurement program ……................................................................................. 10

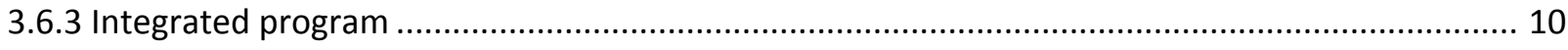

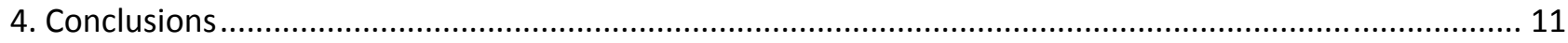

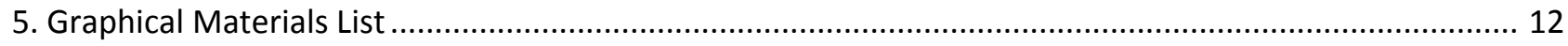

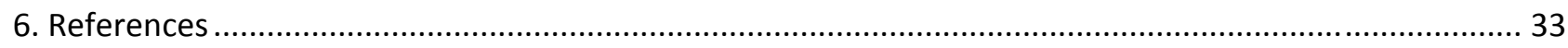

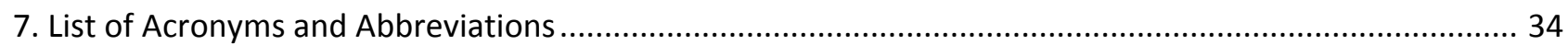




\section{Executive Summary}

This is the final technical report on the program "Optical Fiber Sensor Instrumentation for Slagging Coal Gasifiers", funded by the National Energy Technology Laboratory of the U.S. Department of Energy, and performed by the Center for Photonics Technology (CPT) of the Bradley Department of Electrical and Computer Engineering at Virginia Tech and summarizes research achievements over the entire project period.

The objective of this program is to develop an optical fiber sensing system to monitor refractory thickness and temperature as needed in a slagging coal gasifier. We have demonstrated a system to measure thickness using a high spatial resolution optical time-domain reflectometer (OTDR), and integrated a whitelight interferometry based, surface mounted sapphire temperature sensor into it to realize simultaneous monitoring of thickness and temperature.

For thickness measurement, silica singlemode optical fiber is used. The narrow laser pulse is launched into the fiber from the OTDR and the light partially reflected at a reference point before the light reaches the fiber far end where the second reflection is generated. The temporal separation between the two returned pulses then allows accurate determination of the geometrical distance between the two light reflecting points given the known effective index of refraction of the fiber. To demonstrate refractory wall thickness measurement, a section of the fiber between the two reflections is buried into a refractive brick, which is then either corroded or saw cut to shorten the fiber. At the same time, the brick is heated to different temperatures to demonstrate the capability of high temperature operation. The temperature is increased from room temperature to $600{ }^{\circ} \mathrm{C}$ and then to $1000^{\circ} \mathrm{C}$. For temperatures below $800^{\circ} \mathrm{C}$, we can directly measure the length of the fiber without any problem encountered. However, for higher temperatures, blackbody radiation starts to saturate the OTDR. In this case, a band pass optical filter centered to the OTDR laser wavelength is used and other measures are taken to suppress the blackbody radiation effect. For a saw cut fiber end, an accuracy of $\pm 2 \mathrm{~cm}$ at $1000^{\circ} \mathrm{C}$ is obtained while an accuracy of $\pm 3 \mathrm{~cm}$ at $900^{\circ} \mathrm{C}$ with sodium carbonate corroded fiber is achieved.

For temperature measurement, a single-crystal sapphire fiber based temperature sensor is used, which consists of a C-plane sapphire wafer, a C-plane sapphire fiber, and a ceramic tube to bond the two. One end of the sapphire fiber is inserted into the tube to contact the sapphire wafer and the other end is spliced to a multi-mode fiber using a special splicing method developed at Virginia Tech. Broadband light from a light emitting diode (LED) is launched into the sapphire fiber via the silica multimode fiber and is reflected in part at the two sapphire wafer surfaces. The two reflections would interfere with each other and form an interferometric fringe pattern in the detected optical spectrum from which the optical thickness of the sapphire wafer can be determined. Since the wafer optical thickness is dependent of temperature and both the sapphire fiber and the sapphire wafer can be used at high temperature, measurement of high temperatures well above $1000{ }^{\circ} \mathrm{C}$ can be realized. By a whitelight interferometric signal demodulation algorithm, we have obtained absolute temperature measurement with an accuracy of $\pm 10^{\circ} \mathrm{C}$, from room temperature to $1500^{\circ} \mathrm{C}$. 


\section{Introduction}

Coal gasification is one of the most promising power generation technologies due to its low emissions (removal of up to $99 \%$ of pollutant forming impurities), high efficiency (more than 50\% compared to $33-38 \%$ for conventional coal-fired boilers), and capability to produce additional fuels such as hydrogen. Refractory monitoring and real-time temperature measurements are needed for improved reliability and advanced process control in the coal fired power plants of today as well as the FutureGen power generation systems of the future. The objective of this program is to develop an optical fiber sensing system to monitor refractory thickness and temperature in a slagging coal gasifier.

In a coal gasifier, most of the fuel is not burned, but is chemically broken down by high temperature and pressure in a limited oxygen environment, producing syngas. Integrated gasification combinedcycle (IGCC) plants then clean the syngas and use it as fuel in a combustion turbine. The heat exhaust from the gas turbine is used to produce steam to drive a steam generator, drastically improving the power generation efficiency. Refractory liners are used in entrained-flow slagging gasifiers to protect the gasifier shell from the severe environment, including extremely high temperatures $\left(1250-1550^{\circ} \mathrm{C}\right)$, large and rapid temperature changes, high pressure (400psi or more), hot corrosive gases, particulate erosion, and molten slag attack. Air cooled entrained-flow gasifiers are typically lined with high chrome oxide material that lasts from three to thirty months [1]. The wear rate is highly dependent on the operating conditions and materials, and refractory life has been identified as the most critical factor limiting availability, a key problem for existing gasification technology. Greater than $90 \%$ availability is considered the requirement for power generation [2]. Replacing a gasifier liner costs up to $\$ 1,000,000$ and requires at least ten days of down time. Online instrumentation to measure refractory wear will allow planning of outages for replacement, and time for plants to bring a second gasifier online.

Accurate and reliable temperature measurement is a major concern for efficient gasifier operation. Operating at too high a temperature will reduce the conversion efficiency and significantly shorten the refractory life, while operating at too low a temperature will cause the molten slag to become viscous, plugging the tap-hole. Current thermocouples only last about 30-45 days, mainly due to corrosion from slag and thermal cycling stresses, and suffer from significant drift. Optical and acoustic pyrometers have also been investigated for gasifier temperature measurements, but are subject to interference due to particle radiation or plant noise, or line-of-sight obstruction.

Optical fiber-based sensing instrumentation has been demonstrated to be especially attractive for the measurement of a wide variety of physical and chemical parameters because of such inherent advantages as 1) immunity to electromagnetic interference (EMI), 2) avoidance of ground loops, 3) capability to respond to a wide variety of measurands, 4) high resolution and accuracy, 5) resistance to harsh environments, and 6) ease of integration into large scale fiber networking systems. Optical fiber-based sensors are particularly well suited for high temperature pressure and temperature measurement because they can be engineered to survive environments with temperatures exceeding $1800^{\circ} \mathrm{C}$ through proper choice of optical fiber waveguides. Fiber sensor heads can be fabricated from sapphire, glass, quartz or diamond. Sapphire's physical strength, chemically inert characteristics, and optical properties make it the most suitable candidate for use in very hostile environments. 


\section{Approach}

In this three-year program, the Center for Photonics Technology proposed to develop a fiber optic sensing system for slagging coal gasifiers. A silica-based fiber sensor head, suitable for operation up to $800-900^{\circ}$ will be utilized for monitoring the refractory wall thickness and a sapphire-based fiber sensor head provide temperature data from inside the gasifier. The proposed program builds directly on several key technologies developed at Virginia Tech, including a novel white light interferometry data processing algorithm, silica-to-sapphire fiber connectorization, and sapphire-to-sapphire material bonding.

\subsection{Refractory thickness measurement}

A length of a silica fiber embedded in the refractory wall can be used to monitor the wall thickness as both are corroded by the gasifier environment. It is well known that OTDR can be used for distance measurement and fault locating. We propose to use this method to monitor the corrosion rate through measurement of the embedded fiber length. This scheme is conceptually illustrated in figure 1 .

A pulse of light is launched into the fiber whose end is exposed to the corrosive environment. Some light will be reflected at the fiber end and collected by the detector inside the ODTR. The fiber length can be determined by measuring the time of flight of the light pulse. When the fiber length is shortened due to the corrosion, the fiber length change $\Delta \mathrm{L}$ over a certain time period $\mathrm{T}$ can be obtained and the corrosion rate calculated: $\mathrm{R}=\Delta \mathrm{L} / \mathrm{T}$. The resolution of the distance measure is determined by the light pulse duration and the timing accuracy of the electronic circuits of the OTDR. A high resolution OTDR, such as the one maintained at CPT (Opto-Electronics OFM130), has a spatial resolution of $\pm 5 \mathrm{~mm}$. Because the rate of change by corrosion is slow, we can even further improve the measurement resolution by averaging multiple measurements.

\subsection{High temperature measurement}

The principle of the proposed single-crystal sapphire fiber-based temperature sensor is based on the interference generated by the two surfaces of a sapphire wafer, as shown in figure 2 . The sensing element is the wafer, serving as an extrinsic Fabry-Perot interferometer (EFPI). Reflections at both surfaces of the wafer will interfere with each other, producing a modulated spectrum, whose pattern is determined by the Optical Path Difference (OPD) between the two reflections, or equivalently the wafer's optical thickness (OT, the product of refractive index $n$ and thickness $d$ ). Thermal dependence of both $n$ and $d$ will make the OPD/OT and thus the spectrum pattern sensitive to temperature variation. Therefore the temperature information will be carried by the reflected spectrum.

A schematic of the system is shown in figure 3, consisting of an 850nm LED source, a multi-mode (MM) 3-dB coupler and an Ocean Optics S2000 miniature spectrometer. Figure 4 shows an enlarged view of the sensor head. A $99.8 \%$ alumina tube is used as supporting structure, to which both a $59 \mu \mathrm{m}$-thick sapphire wafer and a $75 \mu \mathrm{m}$ (diameter) sapphire fiber are bonded by high temperature alumina adhesive. The sapphire fiber is coupled with a $100 / 140 \mu \mathrm{m}$ MM silica fiber which is connected to the white light system. Light from the LED travels through the 3-dB coupler 
to the sensor head and is reflected. The reflected signal propagates back to the spectrometer, from which the spectrum data is retrieved and processed by a computer. When temperature varies, the interference signal from the wafer will also change. By monitoring this spectrum shift, the temperature information can be demodulated.

\section{Results and Discussions}

One key component of the thickness measurement system is the OFM 130 OTDR by OptoElectronics, which unlike traditional OTDRs used in telecommunications, offers exceptional spatial resolution $(<10 \mathrm{~mm})$. First several methods were investigated for the fabrication of a reference reflector required to have a strong reflection yet low transmission loss for effective self-calibrated thickness measurement. Then we designed a Matlab program to control the OTDR, acquire data from the OTDR and calculate the thickness. On the temperature measurement, an Ocean Optics fiber spectrometer is used to measure the optical spectrum of the light returned from the sapphire wafer. A program was previously developed for fiber whitelight interferometric systems based on such a spectrometer, but the program is not compatible with the Matlab program for the OTDR system. Therefore, a new software program in Visual Basic has to be developed to integrate the two systems into one single graphic user interface (GUI). In the following sections, each part of the above-mentioned work will be discussed in detail.

\subsection{Thickness sensor fabrication}

To measure the length of fiber, the two reflections with a good signal to noise ratio (SNR) are desired. The reflection from the fiber far end, as shown in figure 5 , is decided by the corrosive environment to which the fiber is exposed, thus is not controllable. However, the reflection from the reference point can be designed carefully for the best system performance. On the one hand, a strong reflection from the reference point is desired for the accurate measurement of this position; on the other hand, the optical loss at the point is supposed to be as small as possible in order to get more reflection from the fiber end so an optimal trade off has to be made. During the project period, we tried several methods to fabricate a good reference point. The following sections give a description of each method investigated.

\subsubsection{Misaligned splicing}

In the beginning, the reference point was made by misaligned splicing. Figure 6 gives the fabricating procedures and a fabricated reference point in this manner is shown in figure 7.

The misaligned splicing method is not easy to control. The misaligned interface has very limited reflection partially because of the very small refractive index difference between core and cladding. This method generates very little reflection while causing significant optical loss, which gives a poor signal to noise ratio not only to the reflection at this point but also the signal from the fiber far end so this method is not adopted for the final system implemention.

\subsubsection{Tubing alignment}

This new method used a capillary tube to align two single mode fibers, as shown in figure 8 . The inner diameter of the capillary tube is $127 \mu \mathrm{m}$, slightly larger than standard single mode fiber whose diameter is $125 \mu \mathrm{m}$. To fabricate a sensor of this type, we simply cleaved two single mode fibers and 
insert them into the capillary tube until they barely touched each other.

When the two fibers are sufficiently close to each other, the transmission loss became very small. Since the reflective index difference between the air and the glass is more than two orders of magnitude larger than the misaligned splicing method, thus a very strong reflection from the junction point can be generated.

\subsubsection{Hollow core fiber}

Another method investigated is to splice a very short length of hollow fiber between two single mode fibers. The idea behind this is similar to the tube method above. Figure 9 is a picture taken from the splicer before splicing the hollow fiber to a second single mode fiber on its right. A hollow fiber with an inner diameter of $10 \mu \mathrm{m}$ (by Polymicro) was used in our experiments. A hollow fiber with a length of around $50 \mu \mathrm{m}$ can be spliced between two single mode fibers easily. The reflection is almost the same as the tube method, but the transmission loss of this hollow fiber is slightly larger.

\subsubsection{Summary of sensor fabrication}

There are also other ways to introduce the reference point required for the distance measurement. For example, by cleaving one arm of a 2 × 2 coupler and place sensing fiber on the other arm, one could also produce a decent reference point. Compared with the other methods, the tube alignment method has advantages of simplicity, low cost, and best reflection intensity to loss ratio, thus was chosen for sensor fabrication in this project. Later results were obtained based this sensor structure unless indicated otherwise.

\subsection{Thickness measurement software}

The software program is composed of three parts: 1) user interface, 2) data acquisition interface between the OTDR and a computer and 3) data processing. Through the user interface, the user can control the data acquisition process, switch between different data processing methods and record original data from the OTDR and computation results from the software. Several parameters such as sample interval, average time and average type can be adjusted. The data acquisition interface between the OTDR and the computer reads data from the OTDR; converts it to the proper format and then transfers the data to the computer for further analysis. The data processing part of the software has a high efficiency algorithm for peak searching, distance measurement and the statistical functions for data analysis. The algorithm for this part is highly intelligent and noise immunized. It can precisely determine where the peak starts and where it ends. The rectangles displayed in figure 10 and figure 11 are generated by the algorithm to indicate the positions of the peaks.

The reflection signal amplitude from the sensing point of the fiber can vary in a large dynamical range. In order to solve the problem caused by the large dynamic range of the reflection signal and the intensity difference between the reference point and sensing point reflection signals, the built-in software algorithms are designed to have the ability to analyze the quality of the signal and automatically adjust the sensitivity level of the OTDR when the signal level is either too low or when the signal is saturated.

During each measurement, the software will first adjust the sensitivity of the OTDR to find the reference point. Then it will adjust the sensitivity again to find the sensing point. These two steps 
are shown in figure 10 and figure 11 . These solve the problem caused when the intensity difference between the two reflection signals (reference point and sensing point) are so large such that a single sensitivity setting for the OTDR cannot properly acquire the two signals at the same time. After finding both peaks, the software will measure their position using the corresponding sensitivity setting and calculate the distance between the reference point and sensing point. This step is illustrated in figure 12.

For on-line real time monitoring, some modifications were made to the above program as the project went on. An axis was added to display the thickness measurement history, providing an instant view of the variations in the fiber length (See figure 13). The upper plot is the same as shown from figure 10 through figure 12, which is designed to display OTDR reflection spectrum. The lower plot in figure 13 is an added axis which is to display all measured distances samples. Also, a red light is added above the "POWER" button to indicate the communication between the OTDR and the computer. If the OTDR is connected, the light is red; otherwise the red light disappears.

\subsection{Thickness measurement results}

\subsubsection{Fiber length measurement at room temperature}

The very first measurement taken was at room temperature with cleaved fiber end. The reference point " $A$ " is fabricated by tube alignment and the end of fiber " $\mathrm{B}$ " is a cleaved end, as seen in figure 14. The origin of the fiber, the reference point and the fiber end point are denoted as $O, A, B$ respectively. The length of the fiber to be measured is marked as " $a$ ". As the fiber end " $\mathrm{B}$ " is cleaved in this case, the reflections at both the reference point and the fiber end are strong and thus distance samples have a very small standard deviation. Measurement with this setup helps us characterize our measurement system and identify the best accuracy we could get. A typical set of distance samples obtained is shown in figure 15.

In this experiment we acquired about 200 samples; the average sampling rate is around 1 minute per sample. From figure 15 we can see that distance samples fluctuate within a range of $4 \mathrm{~mm}$ only with a standard deviation of $0.63 \mathrm{~mm}$. If one averages every 5 samples (corresponding to a data frequency of every 5 minutes), the resulting standard deviation becomes $0.34 \mathrm{~mm}$. The samples in the above experiment fluctuate within a range of $4 \mathrm{~mm}$, which is very close to the OTDR spatial resolution $(5 \mathrm{~mm})$. Thus we know from this single experiment that the software program is excellent because it does not cause any additional loss in accuracy.

\subsubsection{Fiber length measurement at $600^{\circ} \mathrm{C}$}

\subsubsection{Sensor packaging and device setup}

The first step in high temperature tests is to design a protection probe with sensor inside. The fiber was placed into an alumina tube and the tube was partially filled with high temperature glue. The purpose of partial filling was to let air escape when the temperature inside increased. Otherwise the thermal stresses could break the fiber. The packaged fiber was placed in a furnace, as shown in figure 16, and then connected to the OTDR.

\subsubsection{Distance measurement with cleaved fiber end}

First, a well-cleaved fiber was installed in the furnace and heated to $600^{\circ} \mathrm{C}$. A 24-hour test was 
performed whose results are shown in figure 17. The test lasted about 25 hours. During this test, 1574 samples were obtained. The sampling rate was about 1 sample/minute. The standard deviation for the samples was $0.58 \mathrm{~mm}$. If averaged every ten samples (corresponding to 10 minutes), the standard deviation for the averaged length would be $0.27 \mathrm{~mm}$. This 24 -hour test confirmed that the fiber sensor made by the tubing alignment method has a good reflection quality and thus the fiber length can be measured accurately at $600^{\circ} \mathrm{C}$.

\subsubsection{Distance measurement with saw-cut fiber end}

To mimic the corrosion in the gasifiers, we used an electric saw to cut the tube containing the fiber. With a saw-cut fiber end, the reflection intensity from the fiber end can be 10 to $20 \mathrm{~dB}$ less than that of cleaved one. In figure 18, the results of another 24-hour test for a fiber with saw cut end are shown. The $x$-axis represents the hours after startup and the $y$-axis indicates the fiber distance in unit of meters. The distance measured is stable for short periods (i.e., several hours), but in the long term, the distance drifts. In this test, 1484 samples were obtained at a rate around 1 sample/minute. The standard deviation for all the samples is $0.88 \mathrm{~mm}$ and after averaging every 10 samples is $0.76 \mathrm{~mm}$. Compared with a cleaved end, the standard deviation increased. The reason is clearly because of the significantly reduced reflection intensity with a cut fiber end.

\subsubsection{Repeated saw cut experiment}

To further demonstrate the stability and repeatability of the measurement system, six cut and remeasure experiments were performed. Each time the tube was shortened by $3 \mathrm{~cm}$. The results are shown in figure 19. The distance during a single measurement was fairly stable. The interval between two cuts might deviate from $3 \mathrm{~cm}$ a little due to inaccuracy in cutting procedures. From the results, we could assert that the measurement was accurate enough to detect fiber length deduction by $3 \mathrm{~cm}$.

\subsubsection{Fiber length measurement at $1000^{\circ} \mathrm{C}$}

When temperature was increased from $600^{\circ} \mathrm{C}$ to $1000^{\circ} \mathrm{C}$, it was not as easy as for a change from room temperature to $600^{\circ} \mathrm{C}$. One major problem was that the black body radiation started to be a problem for the measurement, more specifically, for the build-in photon detector of the OTDR. Since the OTDR enjoys very high sensitivity, even very little light from the black body radiation could saturate the sensitive OTDR. One important task in $1000^{\circ} \mathrm{C}$ test was to mitigate the black body radiation effect seen by the OTDR.

\subsubsection{Methods to mitigate black body radiation}

As temperature increases from $600^{\circ} \mathrm{C}$, the noise floor seen by the OTDR also increases. At $800^{\circ} \mathrm{C}$, it can saturate the OTDR by $60 \%$. In other words, if the maximum power the OTDR can discriminate is 1.0 , the blackbody radiation lifts the background to 0.6 , as illustrated in figure 20 . At $1000^{\circ} \mathrm{C}$, the blackbody radiation raises the background to 0.99 , a value high enough to bury almost all reflection peaks, as shown in figure 21.

It is clear that measures must be taken to reveal the reflection peak from the saturated OTDR. A band pass filter was therefore used here. The center wavelength of the filter is $1314 \mathrm{~nm}$ with a $5 \mathrm{~dB}$ width of $1 \mathrm{~nm}$, as shown in figure 22 . Figure 23 shows that the built-in OTDR light source has a $5 \mathrm{~dB}$ bandwidth of about $10 \mathrm{~nm}$ and central wavelength of $1308 \mathrm{~nm}$. By inserting the filter into the fiber link, the blackbody radiation-induced background is reduced substantially, to about 0.6 at $1000^{\circ} \mathrm{C}$. Better results could be obtained by using another filter whose center wavelength is $1308 \mathrm{~nm}$ and $5 \mathrm{~dB}$ bandwidth of $1 \mathrm{~nm}$. However due to the availability of such filters at CPT, the $1314 \mathrm{~nm}$ filter 
was used for the remainder of experiments at $1000^{\circ} \mathrm{C}$.

A picture of experimental setup for $1000^{\circ} \mathrm{C}$ testing is shown in figure 24 . Same as in $600^{\circ} \mathrm{C}$ test, the sensing fibers were packaged in an alumina tube loaded into the furnace. A filter was inserted into fiber link between the OTDR and the sensing fibers in the furnace. A GPIB card was used to establish communication between the OTDR and a laptop computer. After the filter was inserted into the fiber link, the black body radiation background decreased so that the previously saturated photo detector could locate the reflection peaks from both the reference point and sensing point again.

Moreover, the background blackbody radiation could be further mitigated by bending the fiber to produce an additional optical loss. In this way, the background can be reduced to a very small value, and the reflection intensity from the fiber end is still sufficient for our measurement. Figure 25 gives a general idea about the fiber bending.

Figure 26 compares OTDR results obtained under different conditions with the same OTDR sensitivity at $1000^{\circ} \mathrm{C}$. Without fiber bending or the use of the optical filter, the black body radiation simply saturates the OTDR. By bending the fiber as shown in figure 25 , the blackbody radiation intensity is reduced (as well as the intensity of reflection peaks) and the first reference point can be seen. If we apply the filter without the fiber bending the blackbody radiation is reduced to about 0.68 , and both reflection peaks appear (Note that the inserted filter extends the length of fiber link, and the position of reference peak is shifted afterwards). With both the filter and fiber bending applied, the radiation induced background is reduced to about 0.2. Both reflection peaks appear as they do in tests at room temperature. Thus the software is able to measure the fiber length without hindrance by the blackbody radiation.

\subsubsection{24-hour stability test}

In this test, the fiber ends were cut with an electric saw (not cleaved to an optical surface). With the setup shown in figure 24 , we performed a 24 hours test at $1000^{\circ} \mathrm{C}$. The results are shown in figure 27. We acquired 1337 samples in 24 hours and 15 minutes; the average sampling rate is around $0.919 \mathrm{~min} / \mathrm{sample}$. Thickness samples fluctuate within a range of no more than $2 \mathrm{~cm}$. The standard deviation for this measurement is $5.1 \mathrm{~mm}$. Using a ten sample average, the resulting standard deviation is $4 \mathrm{~mm}$. However, the sampling rate depends on the stability of the reflection intensity and software parameters; therefore, it may change from test to test. In later experiments, we found that the typical value of standard deviation for raw data is about $1 \mathrm{~cm}$. After averaging every ten samples, the typical standard deviation is less than $5 \mathrm{~mm}$.

\subsubsection{Repeated saw cut experiment}

To mimic the corrosion in the gasifiers, an electric saw was used to repeatedly cut the tube and the fibers. To further demonstrate the stability and repeatability of the measuring system, five cut and re-measure experiments were performed. Each time the tube was cut by $3 \mathrm{~cm}$, but re-splicing the sensor probe to the OTDR each time required both fiber ends to be cleaved, which reduced fiber length by a small additional amount. Repeated experiment results are shown in figure 28 . The actual length shortened for each cut differs, ranging from $10 \mathrm{~cm}$ to $4 \mathrm{~cm}$. The sampling rate also varies for measurement after each cut. Fiber was measured for about three hours after each individual cut, but the number of samples acquired during that time period are different, ranging from less than 100 to 200. A slower sampling rate is caused by faster change of reflection intensity because software program has to adjust OTDR sensitivity to keep the reflection peak value before each measurement is taken. Thus rapid change in reflection intensity would keep software programming adjusting the 
sensitivity of the OTDR instead of taking measurements. Samples measured after each cut fluctuate within a range of $2 \mathrm{~cm}$, generally speaking. The red and green in figure 28 give the fluctuation range while the blue line indicates the average length for each cut. Figure 29 displays the results after averaging every ten samples.

From figure 29, we are confident that the thickness measurement system could tell thickness deduction in the order of $\pm 1 \mathrm{~cm}$. Comparing with results obtained from $600^{\circ} \mathrm{C}$, it can be seen that the standard deviation here is larger than that in $600^{\circ} \mathrm{C}$ test, because of the reduced SNR in the fiber end reflection at higher temperature.

\subsection{Corrosion test for thickness measurement}

Even though we used the saw cut experiments to mimic the deduction of fiber length by corrosion, it was still open to doubt that whether this system can work in presence of a corrosion source. This test was performed to answer this question. Eventually in coal gasifiers, the major corrosion source would be a material named "coal slag". However, it is rather difficult to create such an environment in our lab that can corrode a refractory material with fiber embedded by a substantial length. Sodium carbonate was thus used instead. Sodium carbonate has a melting point of $851^{\circ} \mathrm{C}$, and the corrosion speed is very fast.

In the experiment, a crucible was used to hold sodium carbonate powder in a furnace; the alumina tube containing the fiber sensor was immersed into the sodium carbonate powder, as shown in figure 30. After corrosion, the reflection from the fiber end was weakened and was not visible if the band pass filter was present. Therefore, the filter was removed and the fiber was bent to mitigate the black body radiation, as discussed in section 3.3.3.1.

Before corrosion, the alumina tube and silica fiber were cut using an electric saw. The fiber distance was measured at $900^{\circ} \mathrm{C}$ and the result is shown by the blue line in figure 31 . The measured distance has a standard deviation of $4.4 \mathrm{~mm}$, about the same as previous results. Then the furnace was cooled down and the crucible with sodium carbonate was placed inside the furnace with the packaged fiber immersed into the sodium carbonate powder. The furnace was then heated to $900^{\circ} \mathrm{C}$ again. The rapid corrosion rate, which was much faster than the sampling rate, precluded collection of in situ corrosion data. The thickness measurement samples after corrosion are shown by the green line in figure 31. Comparing the two traces, the average fiber distance was reduced by about $7 \mathrm{~cm}$, and the standard deviation after corrosion increased to $8.2 \mathrm{~mm}$, as a result of reduced SNR for the light reflected from the fiber end by corrosion.

\subsection{Temperature sensor structure and calibration}

A sapphire wafer based temperature sensor consists of a polished sapphire fiber, a C-plane sapphire wafer and an alumina tube to bond the two together. Sensor fabrication starts with polishing the sapphire fiber and the alumina tube, followed by sensor head bonding, and finishes with splicing the sapphire fiber to a silica multi-mode fiber.

\subsubsection{Sapphire fiber connection}

To splice the sapphire fiber to a multimode fiber, the end surface must be as smooth as possible. Thus sapphire fiber was polished using diamond polishing pads with a grain size ranging from 
$30 \mu \mathrm{m}$ down to $0.5 \mu \mathrm{m}$. After $0.5 \mu \mathrm{m}$ polishing, one can expect an end surface similar to the one shown in figure 32. The Center for Photonics Technology at Virginia Tech has developed a method for splicing a sapphire fiber to a multi-mode silica fiber. The sapphire fiber diameter used here is $75 \mu \mathrm{m}$ and the multi-mode fiber diameter is $100 \mu \mathrm{m}$ for the core and $140 \mu \mathrm{m}$ for the cladding. Detailed information on the splicing method can be found in [3].

\subsubsection{Sensor head fabrication}

One end of the alumina tube is polished. Then the sapphire wafer is bonded to the polished end of the alumina tube using a high temperature adhesive. The sapphire fiber is fed into the alumina tube until it nearly touches the sapphire wafer. Finally, the sapphire fiber is bonded also to the alumina tube using the same adhesive. By this step, the sensor head fabrication is finished.

\subsubsection{Sensor testing and calibration}

The optical system configuration is shown in figure 34. The light from the LED is launched into a $3-\mathrm{dB}$ multi-mode fiber coupler and propagates to the sensor head. The reflected spectrum is received by the Ocean Optics spectrometer and is sent to the computer for further signal processing. Because of the inference of the two reflections from the two surfaces of the sapphire wafer, the optical spectrum of the returned optical signal is modulated. The spectrum pattern is determined by the optical thickness (OT) of the sapphire wafer, which is the product of the sapphire refractive index $n$ and wafer geometric thickness $d$. As both parameters are dependent on temperature, the temperature can be determined by demodulating the spectrum. The method used in demodulation is fully discussed in [4]. A representative calibration curve is shown in figure 35.

After calibration, the computer program calculates the accurate optical path distance (OPD), which in this case is the doubled OT due to the round trip of the second reflection in the wafer. By comparing the calculated OPD with the calibration curve above, the program finds the correct temperature. A carefully calibrated sensor could have accuracy around $10^{\circ} \mathrm{C}$. It should be pointed that since the calibration was done by the use of a thermocouple which has a similar accuracy, it is our believe that the actual accuracy of the sapphire fibber sensor may be better than $10^{\circ} \mathrm{C}$.

\subsubsection{Sensor packaging}

To protect the sensor head, we designed the following package using four tubes (Figure 36). From innermost to outermost are alumina, sapphire, alumina and alumina tubes. The largest alumina tube is open at both ends. All other tubes are closed at one end. To bond the tubes together, the gaps in between the three smallest tubes are filled with bulk fiber. At the sensing end, the gap between the two largest alumina tubes is filled with refractory material to bond them tightly. At the other end, the gap is filled with bulk fiber to prevent anything else from entering into the tubes. After packaging, the sensor head is about one inch from the surface of the outermost alumina tube.

\subsection{Integration of two systems}

Separate software programs using different languages and operating independently, were previously used for temperature and thickness measurements. At the final stage of this project, these programs were integrated to provide an easy control and management of both the sensors. The new program developed was able to perform both measurements simultaneously using a single graphical user interface (GUI). 


\subsubsection{Thickness monitoring program}

In the beginning, a software program based on Matlab was developed for thickness measurement. This GUI is shown in figure 37, where two plots are visible. The upper plot shows the OTDR screen, while the lower one shows the calculated distance samples. The y-axis of the upper plot is the normalized reflection intensity seen on the OTDR's screen; its X-axis is the distance from the reference point. From the upper plot it is evident whether any reflection peak is visible within the scope. The program will adjust the sensitivity of the OTDR in order to make an accurate measurement for the thickness, and then the measured results are displayed in the lower plot. The yaxis in the lower plot is the distance in meters; the $\mathrm{x}$-axis is the index of samples measured. There are also some other buttons and input boxes to control the measurement parameters.

\subsubsection{Temperature measurement program}

The software developed at CPT for whitelight interferometry based temperature measurement, in its original form, was developed for field tests at TECO's Polk Power Station in Florida under a separate DOE project. The GUI of this original software is shown in figure 38. On the right side of the figure, the three groups of check boxes correspond to three temperature channels, named S2000Master, S2000Slave and USB2000 respectively. One can choose which channels are enabled for measurement by checking the check boxes to the left of the channel names. For each channel, one can further specify if this channel shall be displayed, calculated or logged. If a channel is enabled and all check boxes for this channel are checked, the program will collect spectra from that channel; calculate the optical path difference (OPD), then log the spectrum data for future reference. The temperature measured by that channel is calculated using pre-calibrated coefficients and displayed in the bottom right corner. The left side of the window has a plot that can display spectra from all enabled channels in different colors. At the bottom there are four buttons for the user to configure channels, start or stop measurement, or exit that program.

\subsubsection{Integrated program}

As previously mentioned, these two programs were written in different languages. Fortunately, hybrid programming techniques are available to combine the two easily. The Matlab programming interface in Visual Basic was used here. The integrated program is still mainly based on Visual Basic. However, the Matlab-based thickness measurement program can be started through the Matlab programming interface embedded in Visual Basic. Figure 39 shows the integrated program. The integrated program looks similar to the temperature measurement program with the exception of a button labeled "Thickness monitors" at the bottom line. When this button is clicked, the Matlab-based thickness measurement program is brought up, as shown in figure 40. These two windows can be dragged and dropped in order to view them simultaneously. The parameters can be changed in each program, but are controlled through a single GUI.

After integration, the new program was tested for both thickness and temperature measurement. They all worked as well as each individual program and the obtained data showed that the accuracy of measurement did not change at all because of the software integration. 


\section{Conclusions}

During the project period, an integrated fiber optic sensor system for thickness and temperature measurements has been developed for potential future applications in a coal gasifier. The thickness measurement based on high resolution optical time domain reflectometry tested at various elevated temperatures up to $1000^{\circ} \mathrm{C}$. An accuracy of $\pm 2 \mathrm{~cm}$ was obtained at $1000^{\circ} \mathrm{C}$ for saw cut silica fiber end. And the accuracy was typically about $\pm 3 \mathrm{~cm}$ as the silica fiber was corroded by sodium carbonate at $900^{\circ} \mathrm{C}$. The sapphire wafer based temperature measurement was tested at temperatures up to $1500^{\circ} \mathrm{C}$. Sensor accuracy was within $\pm 10^{\circ} \mathrm{C}$ after calibration by thermocouples. 


\section{Graphical Materials List}

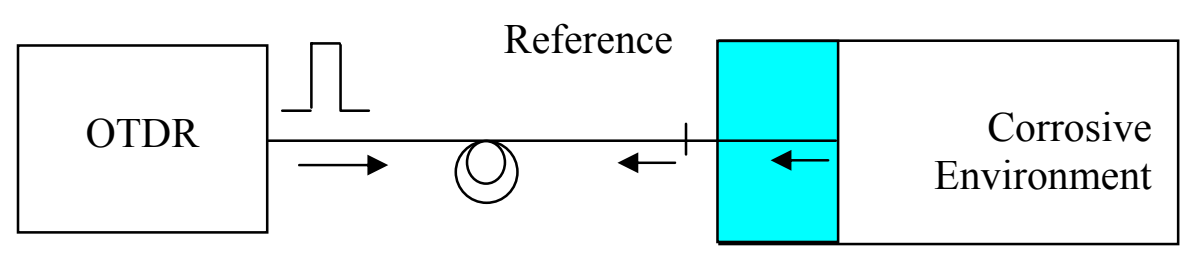

Figure 1: Corrosion rate measurement scheme using an OTDR with a spatial resolution of $5 \mathrm{~mm}$

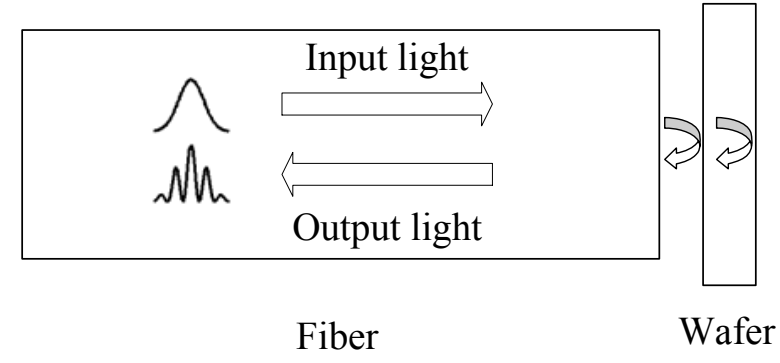

Figure 2: Sapphire fiber temperature sensor principle diagram

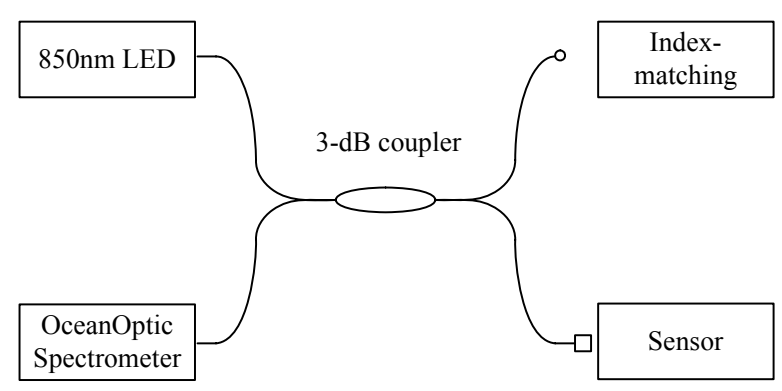

Figure 3: White-light interferometric system diagram

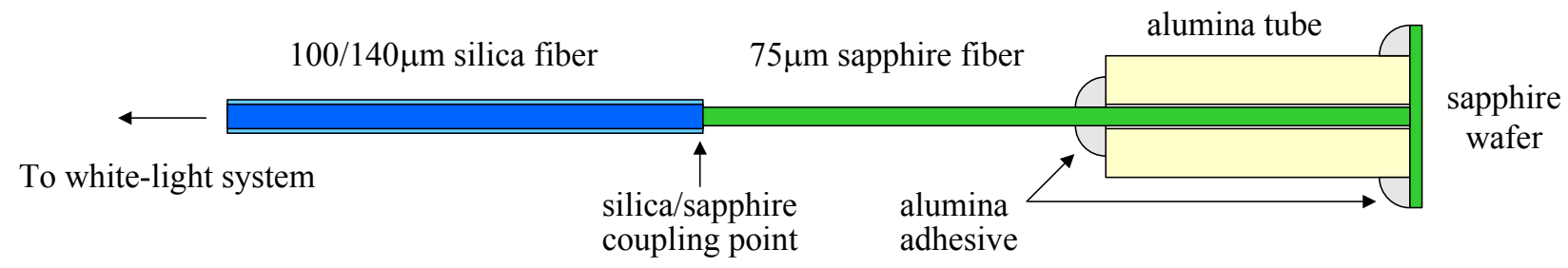

Figure 4: Temperature sensor head structure 


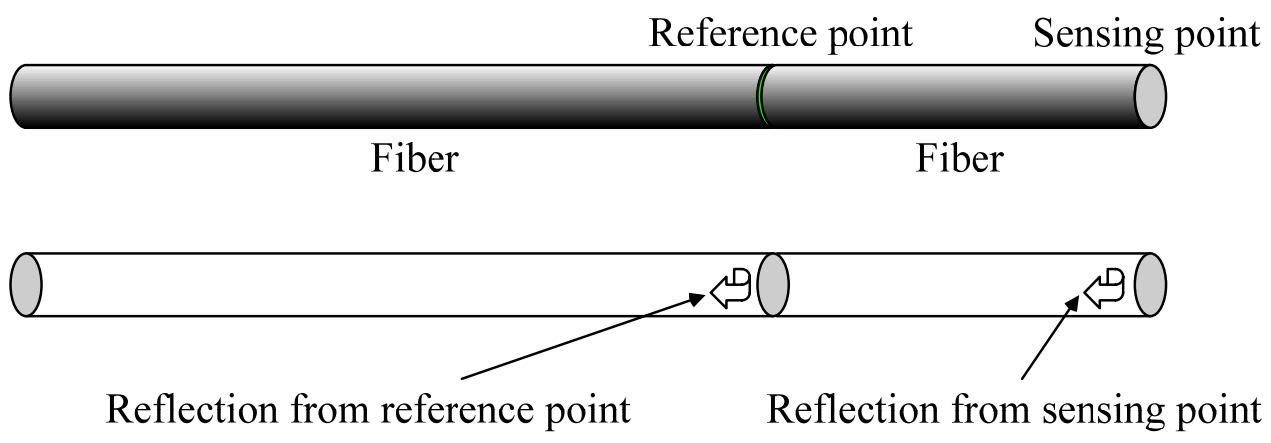

Figure 5: The structure of thickness sensor 


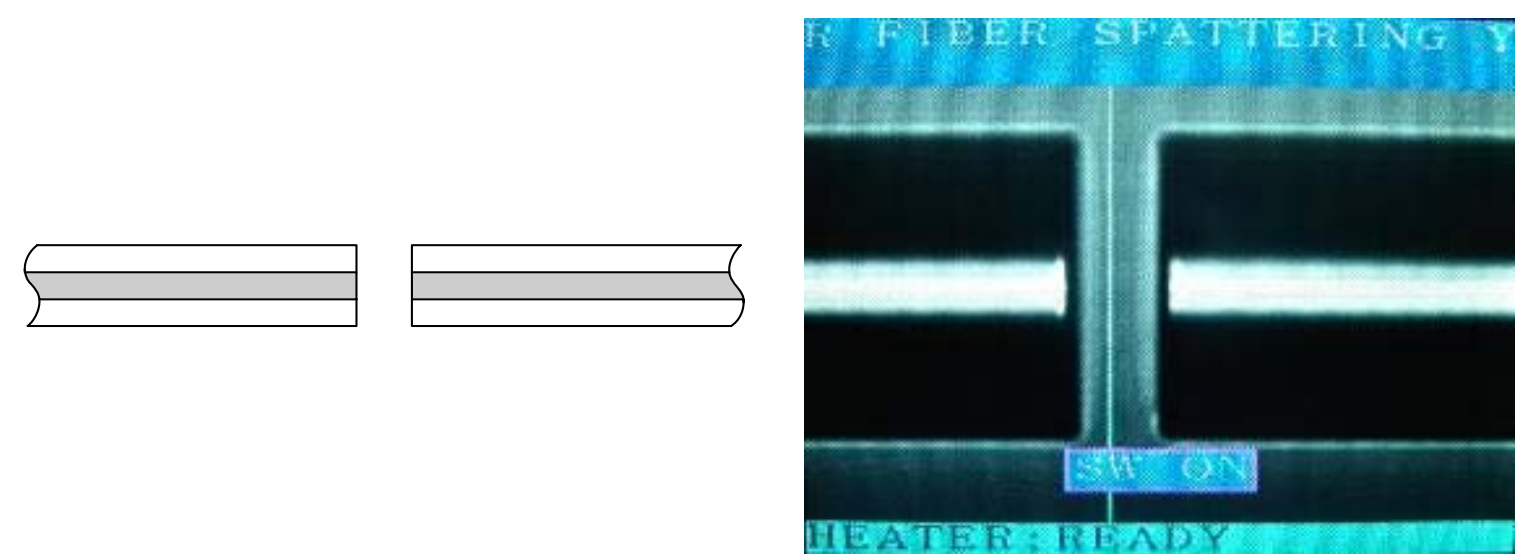

i) Prepare two fibers, each with one cleaved end

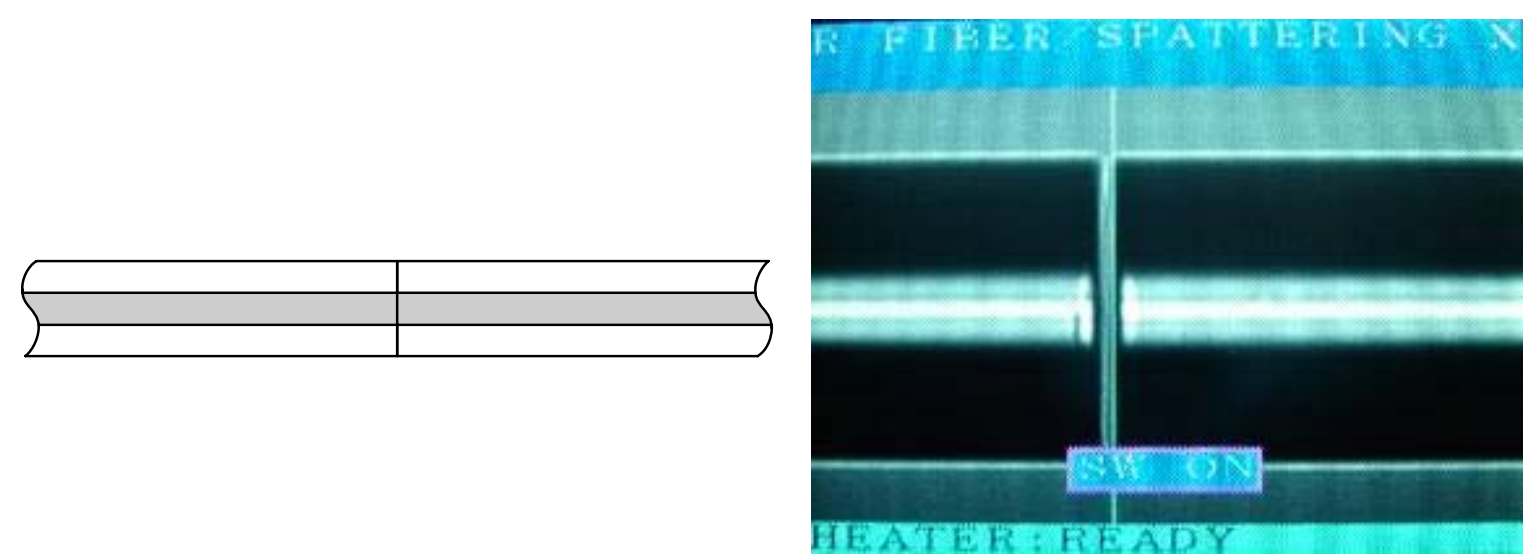

ii) Align the two fibers

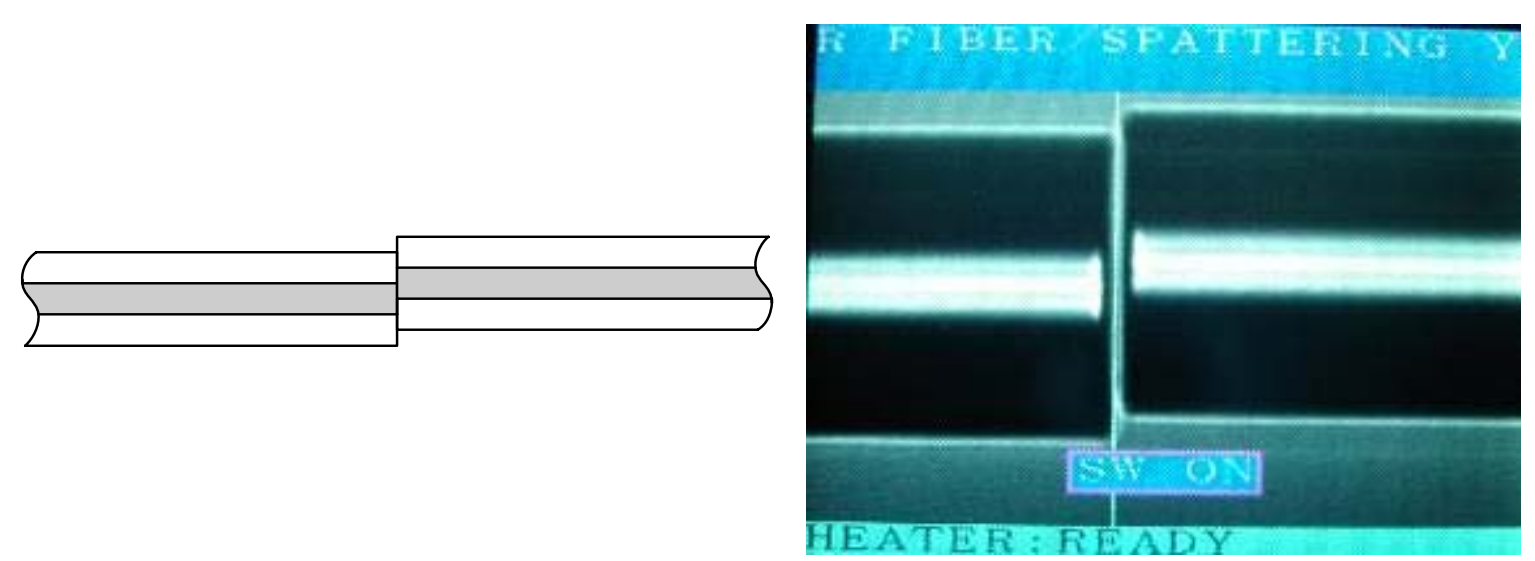

iii) Shift one fiber and then splice

Figure 6: Sensor fabrication procedure 

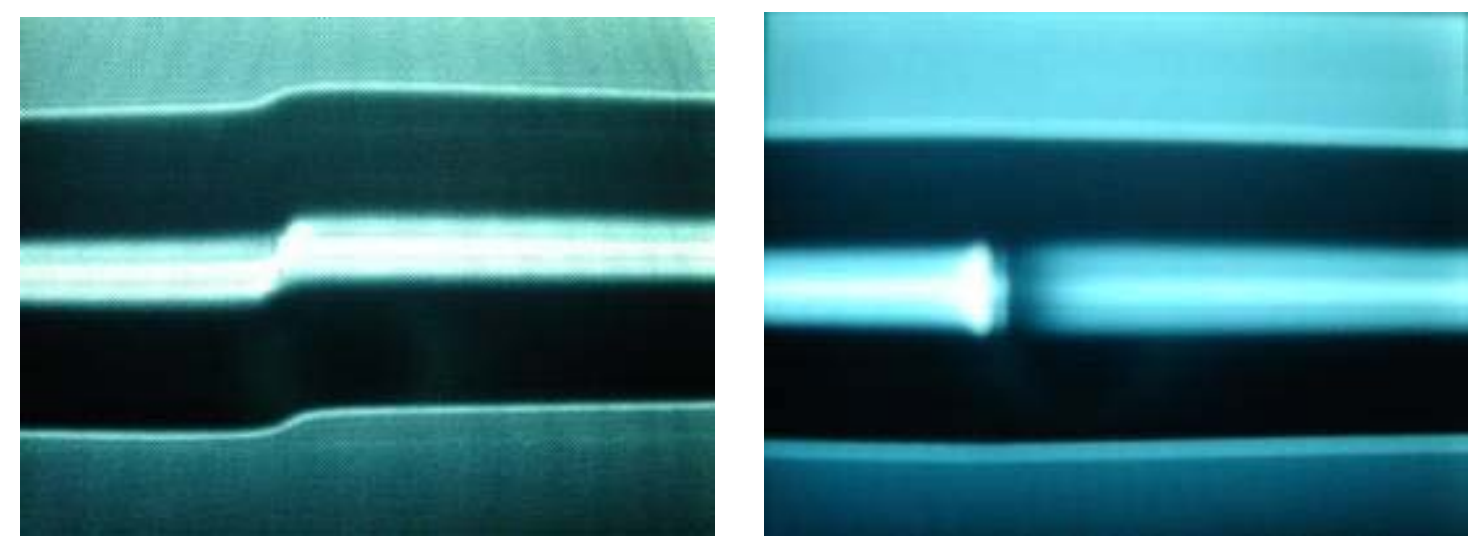

Figure 7: Finished reflection point in the fiber (Photos are taken from different angles.)

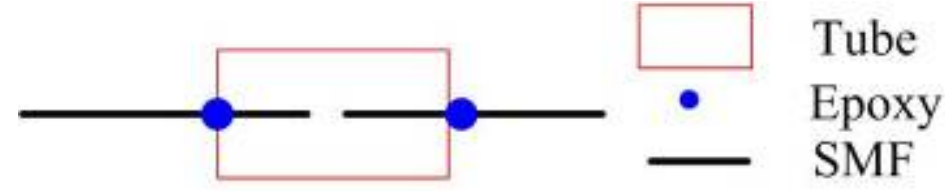

Figure 8: Sensor fabrication of tubing alignment

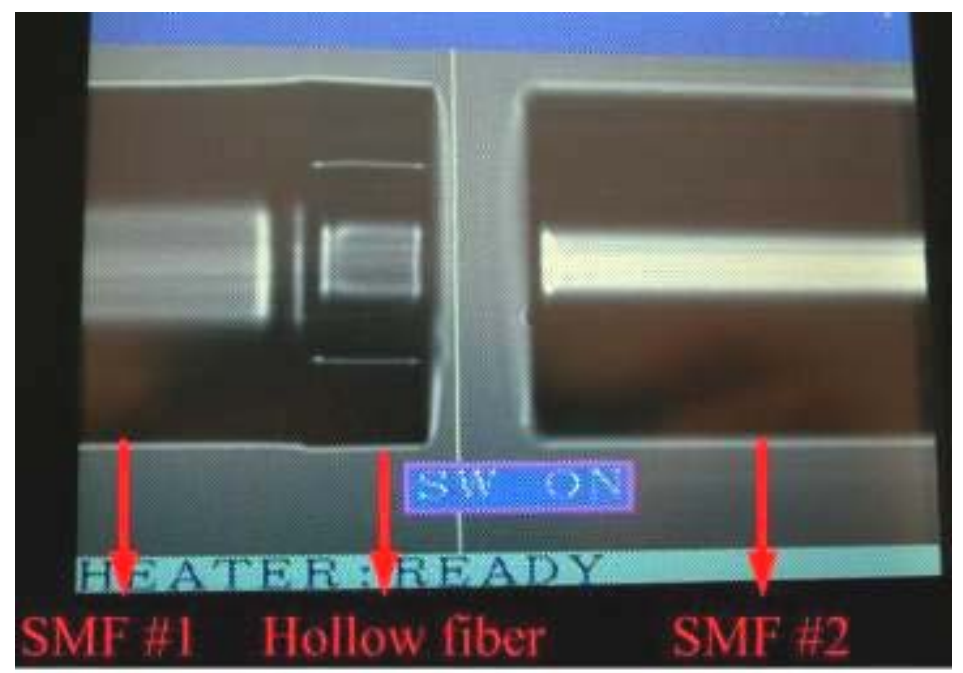

Figure 9: Splice hollow fiber between two single mode fibers 


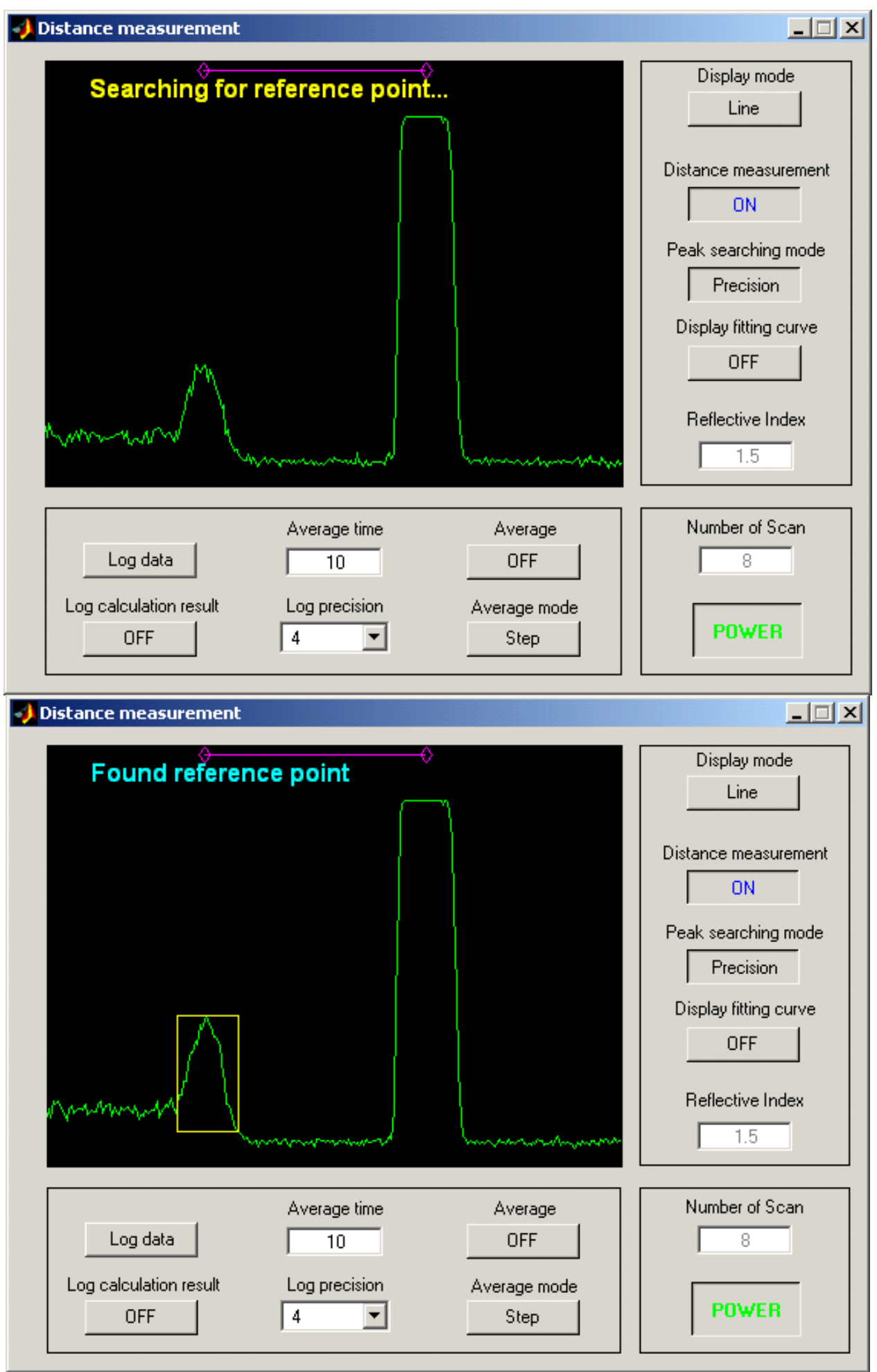

Figure 10: Reference point search procedure 


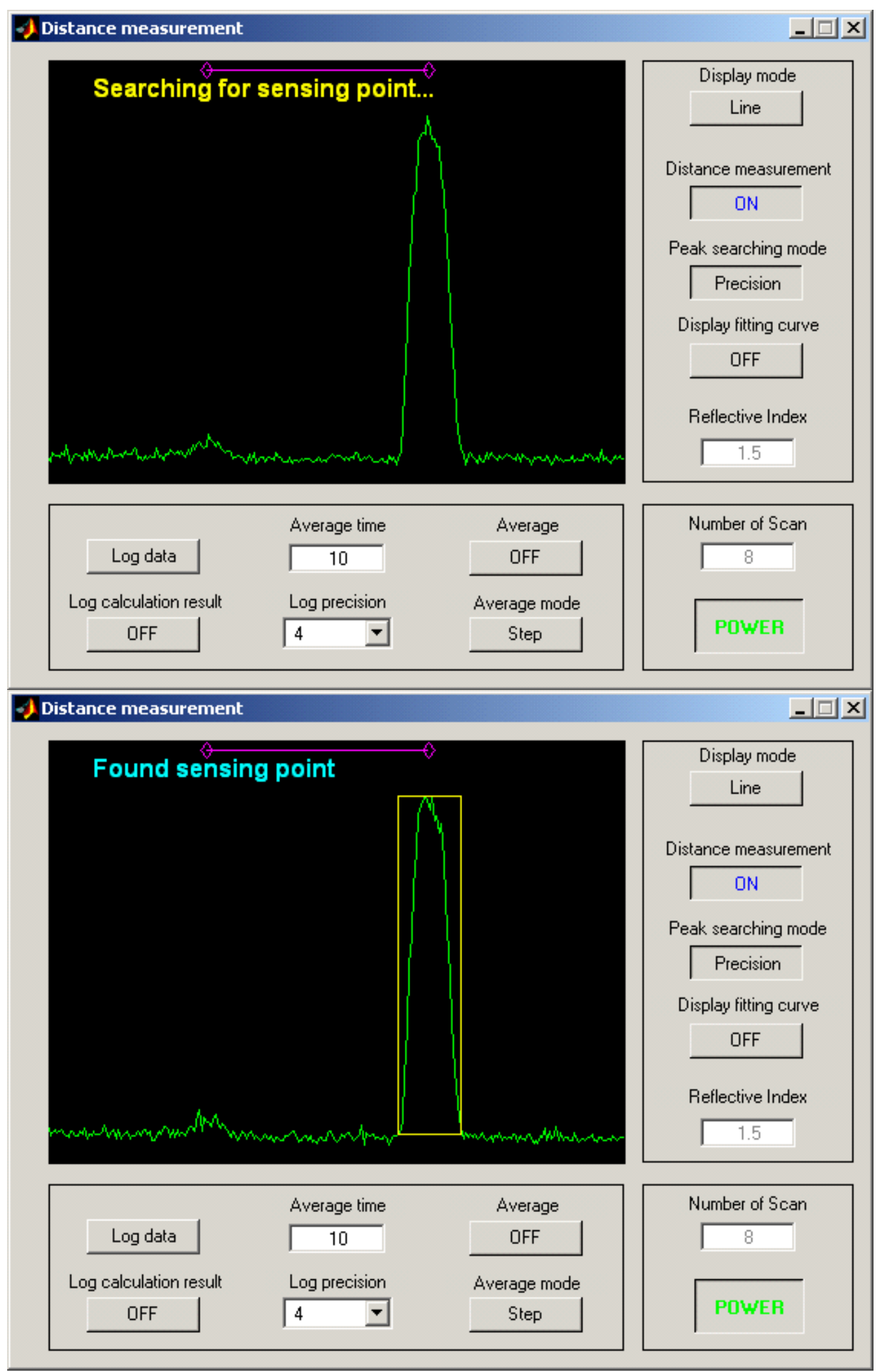

Figure 11: Sensing point search procedure 


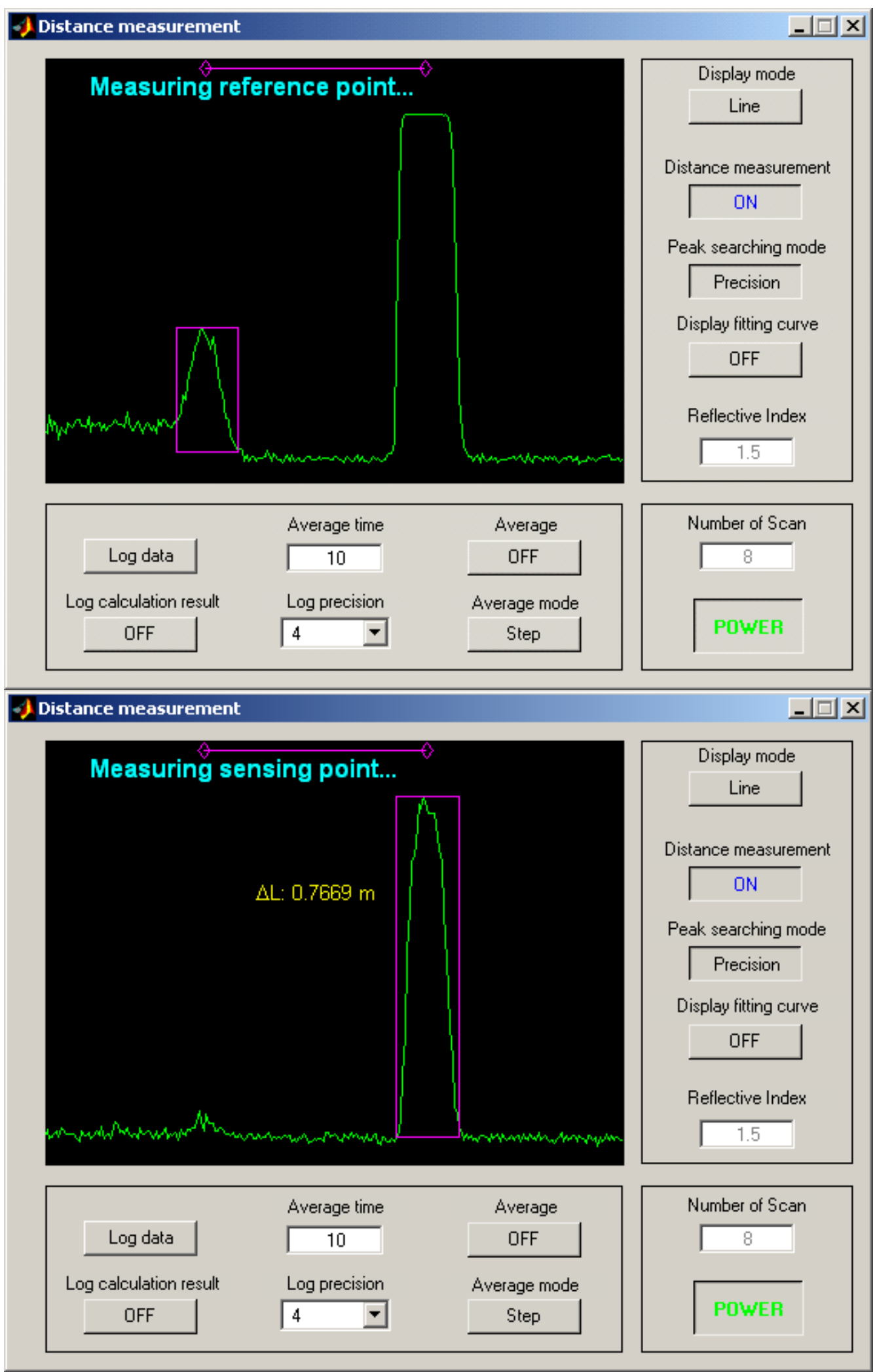

Figure 12: Peak position measurement and distance measurement 


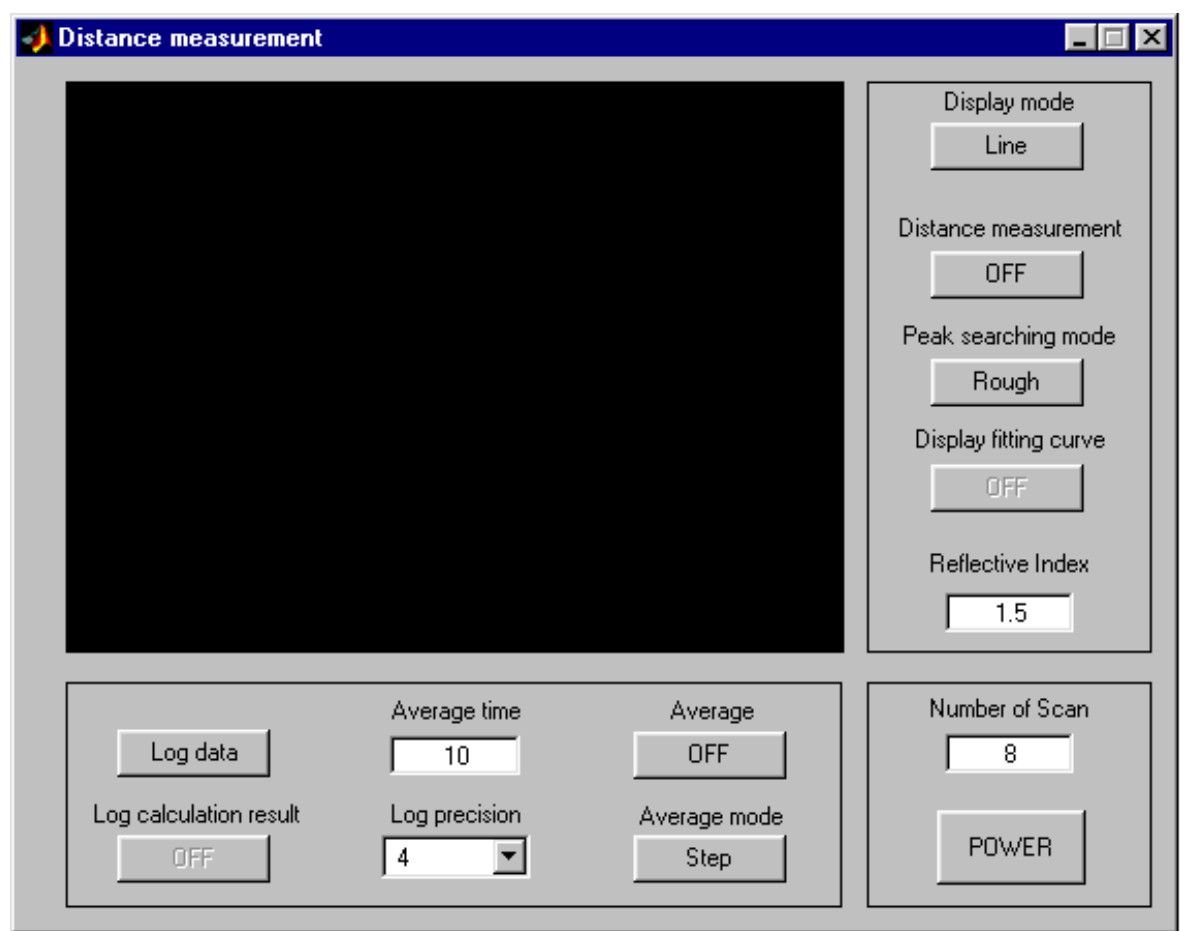

(a) First version of user interface

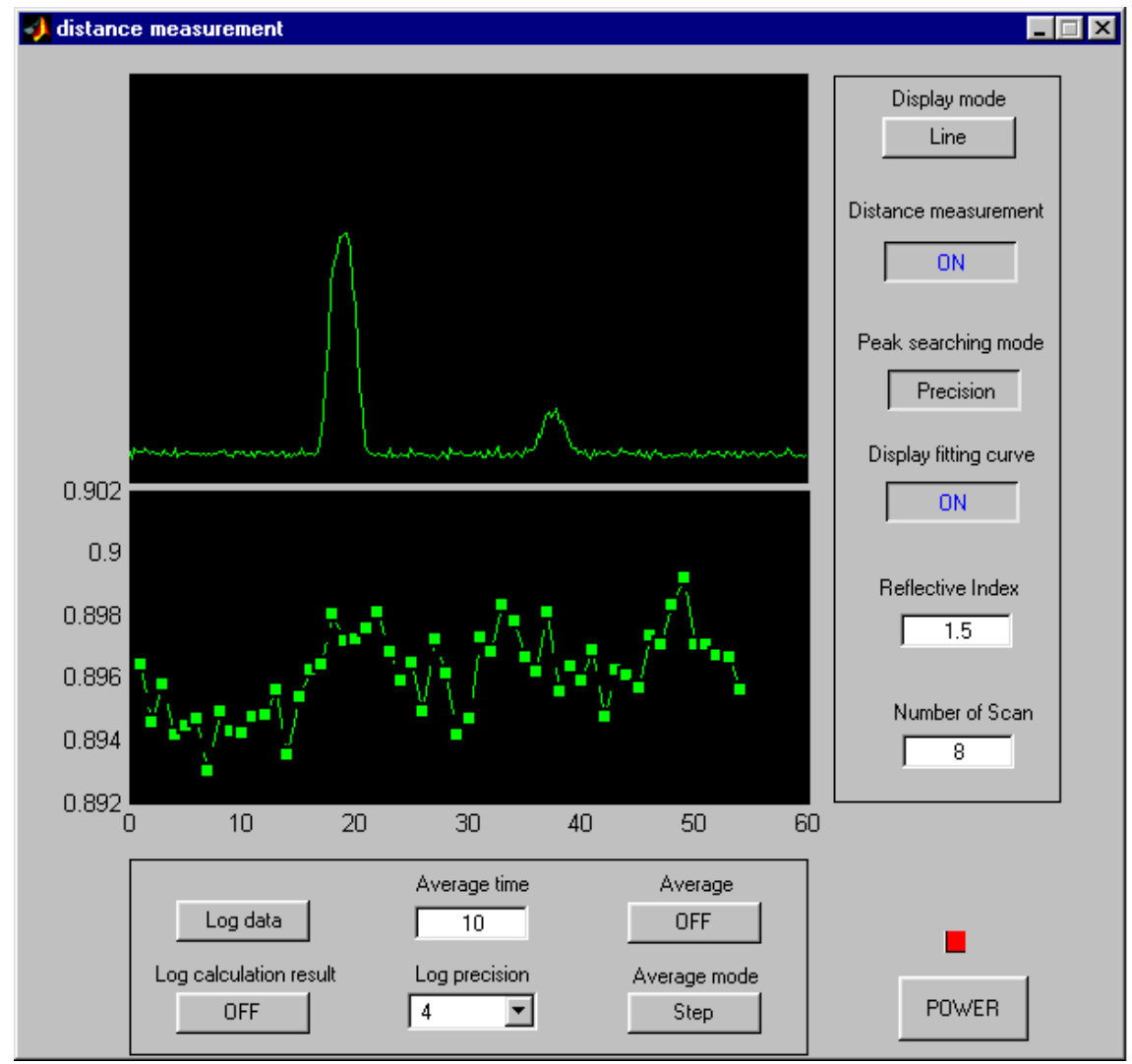

(b) Modified user interface

Figure 13: Additional features were added to the user interface 


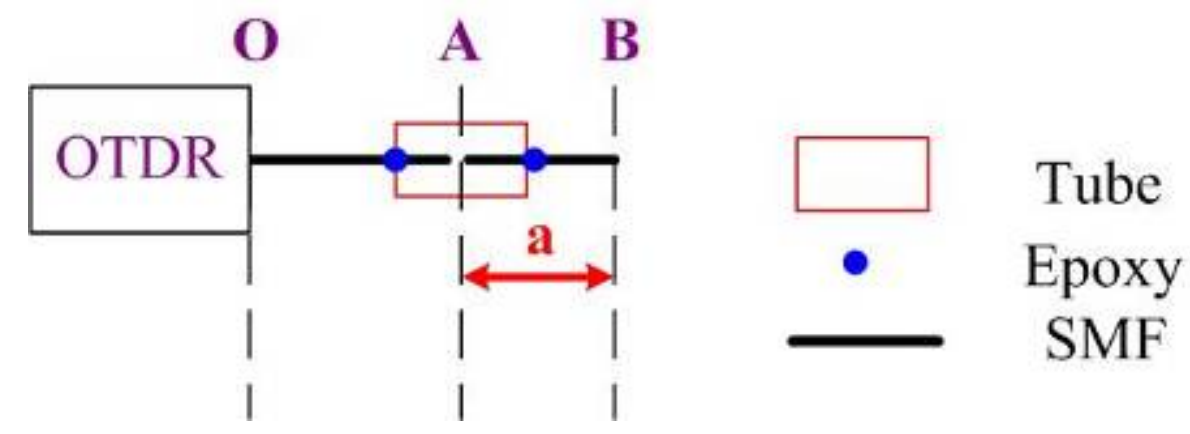

Figure 14: System configuration for distance measurement experiment

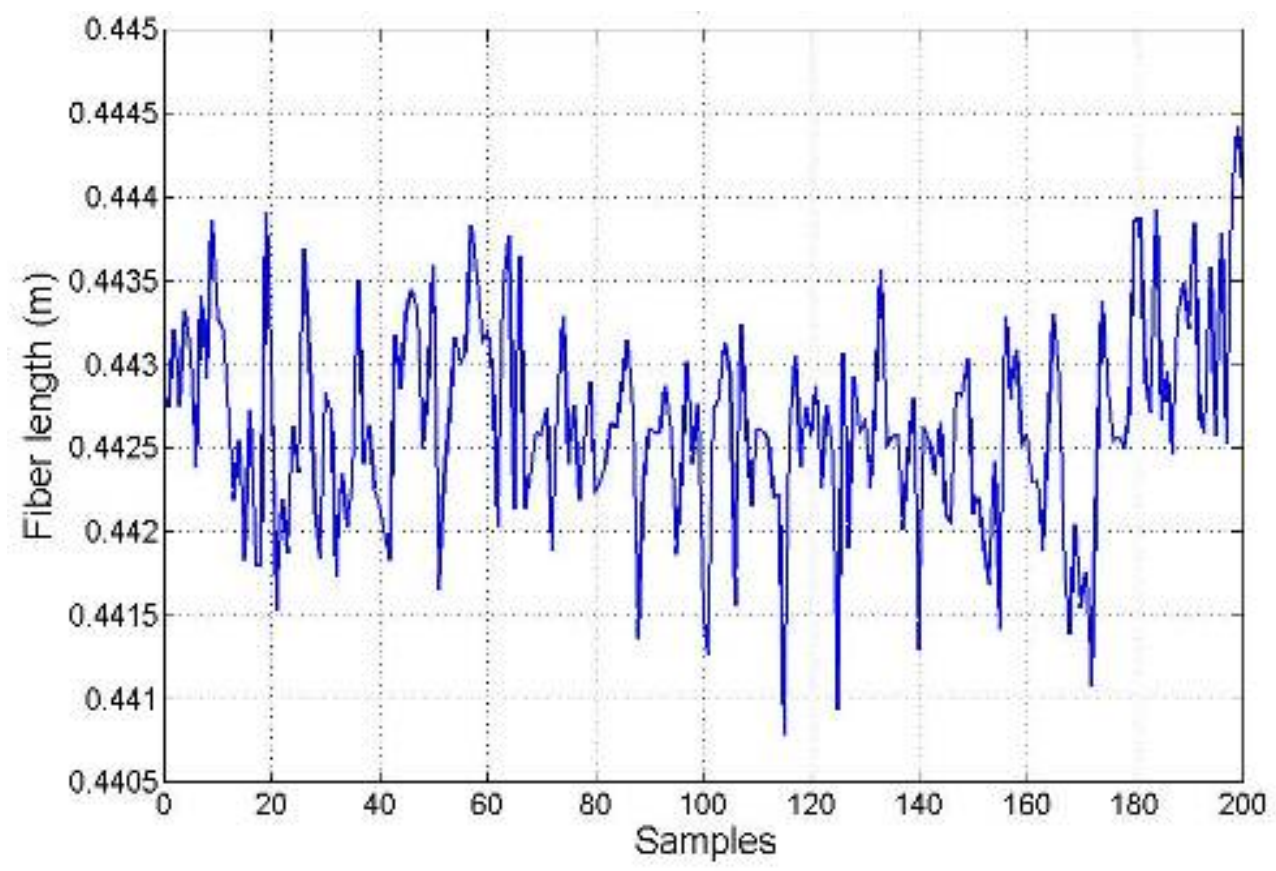

Figure 15: Representative room temperature distance samples of a well-cleaved fiber

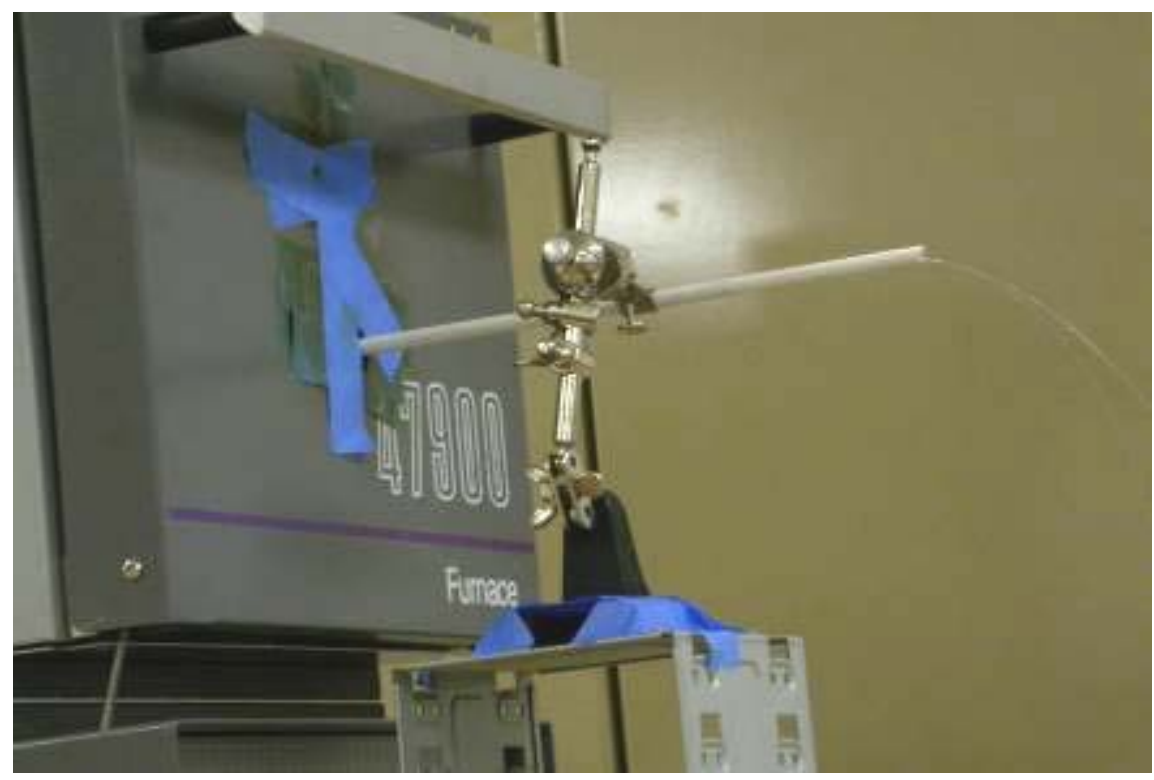

Figure 16: Device setup for high temperature test 


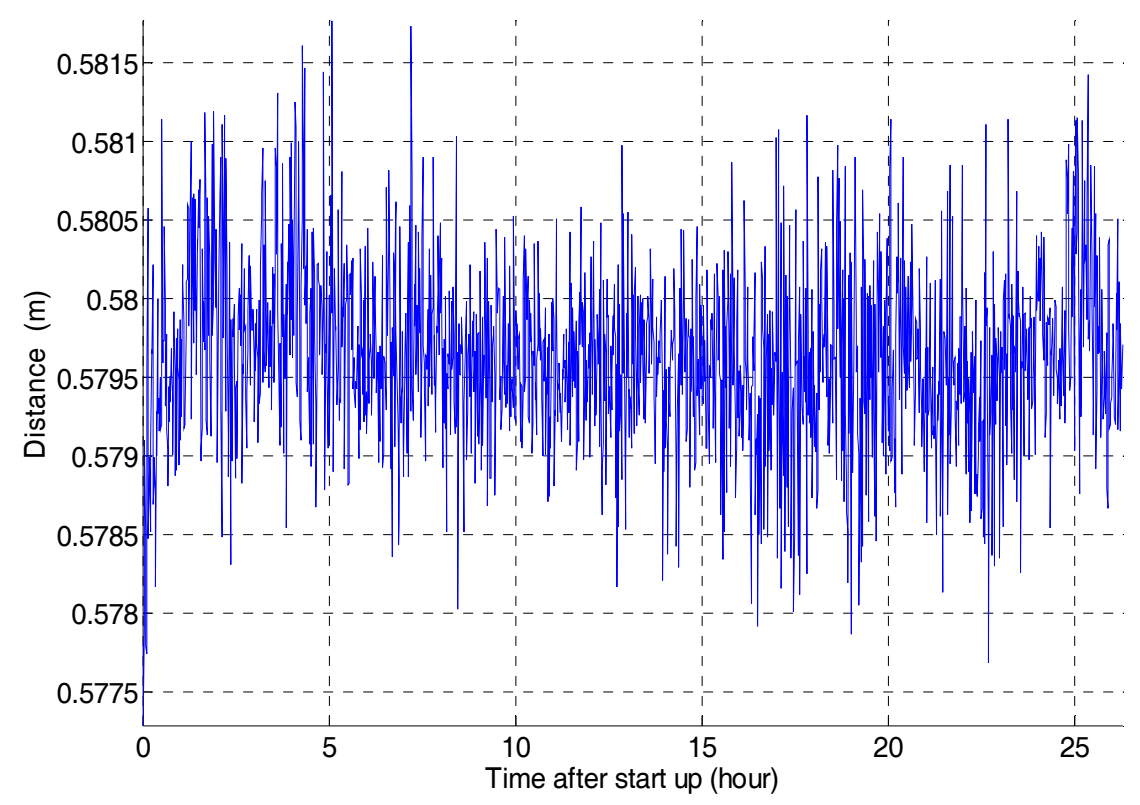

Figure 17: 24-hour test at $600^{\circ} \mathrm{C}$ for fiber with cleaved end

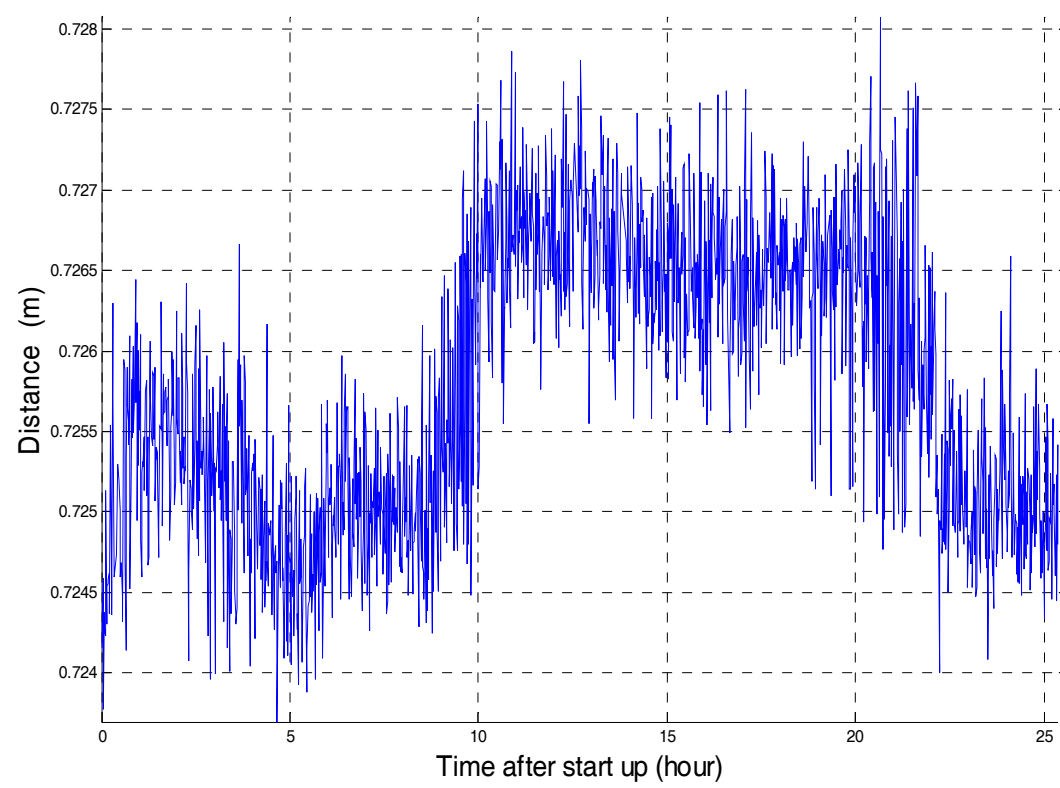

Figure 18: 24-hour test at $600^{\circ} \mathrm{C}$ for fiber with saw cut end 


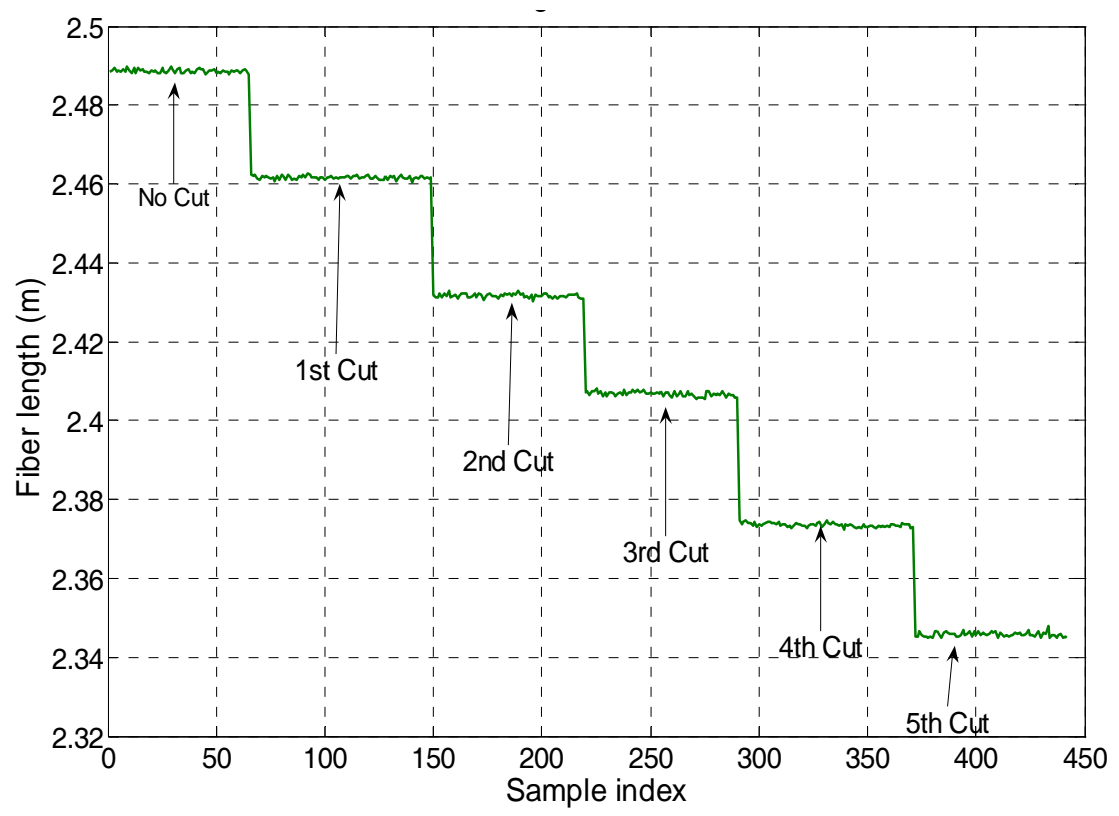

Figure 19: Repeated fiber cut results

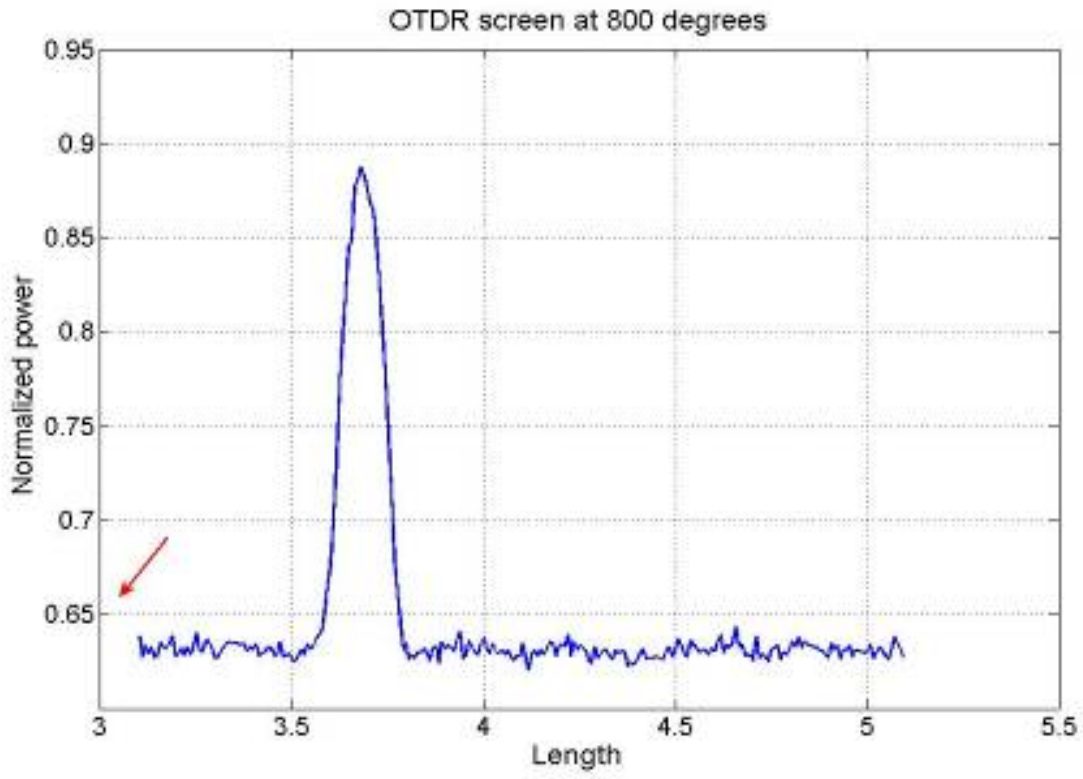

Figure 20: Blackbody radiation seen by the OTDR at $800^{\circ} \mathrm{C}$ 


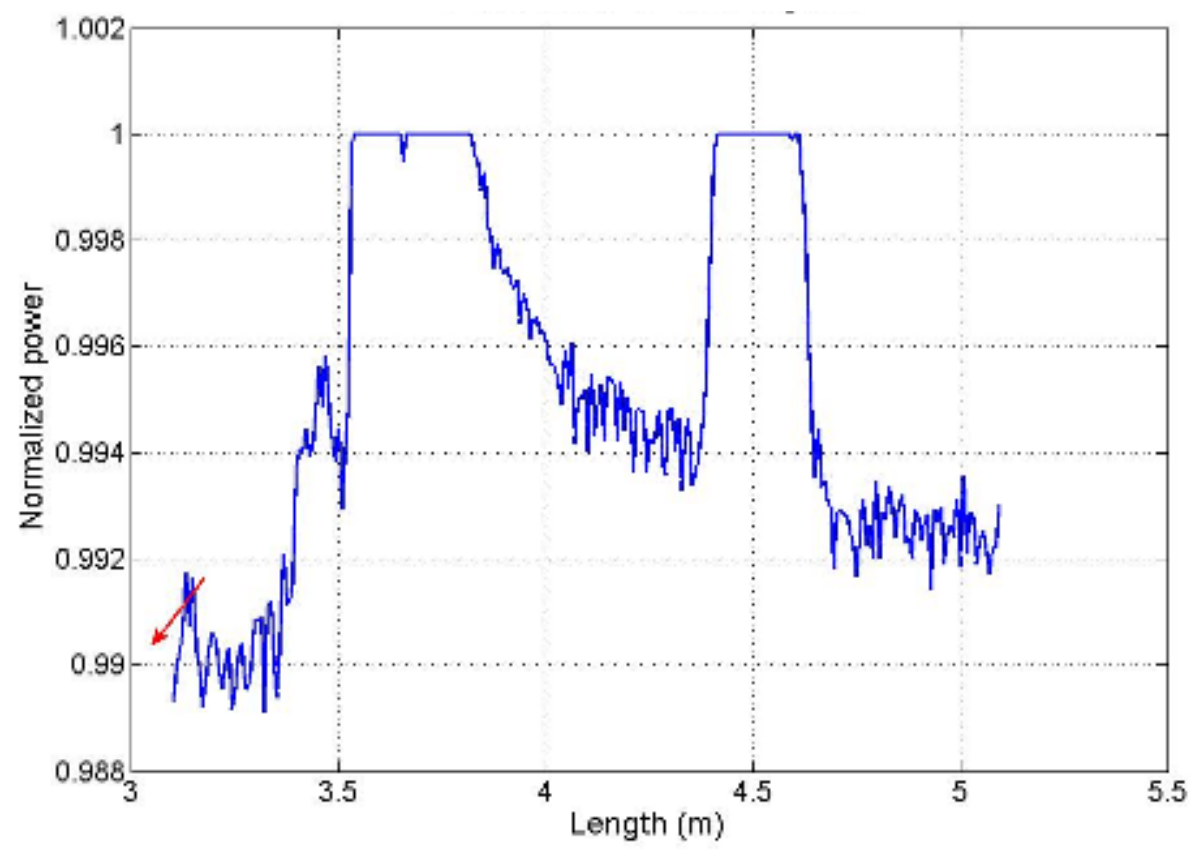

Figure 21: Blackbody radiation seen by the OTDR at $1000^{\circ} \mathrm{C}$

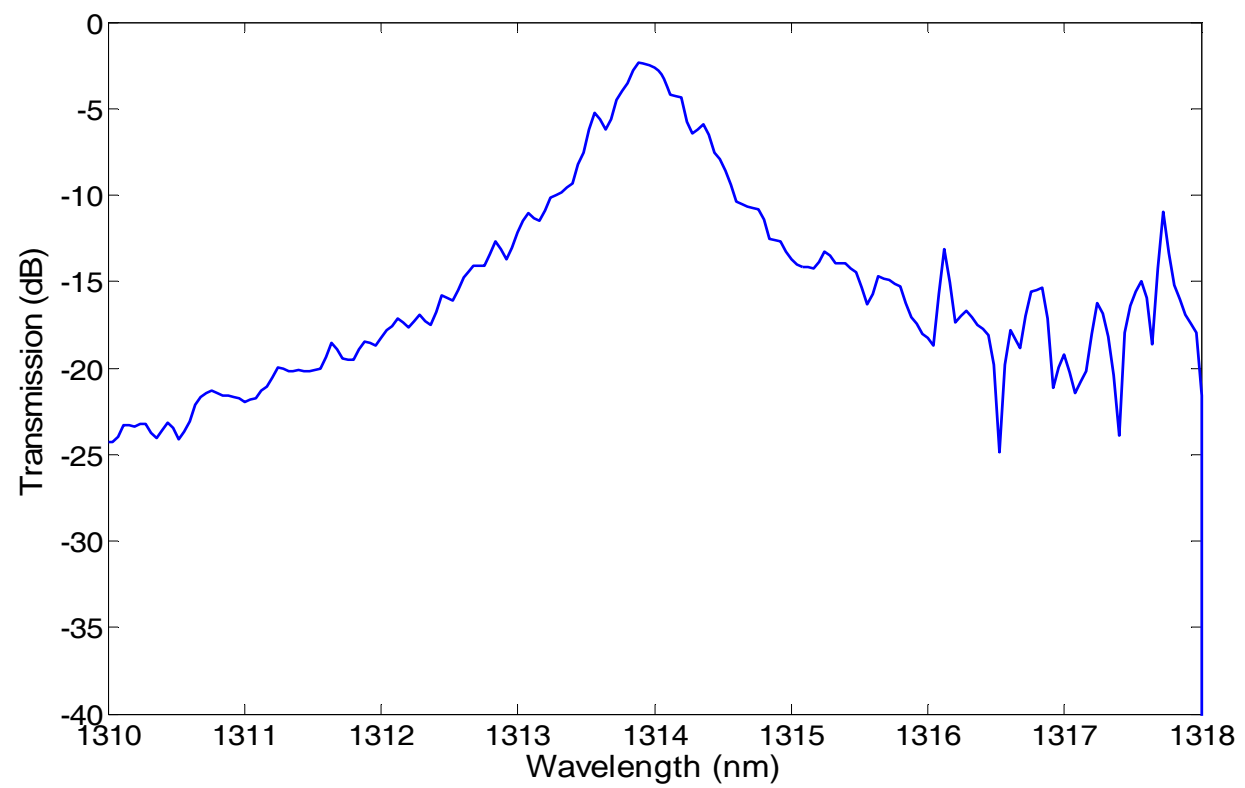

Figure 22: Transmission spectrum of bandpass filter used to mitigate effects of blackbody radiation 


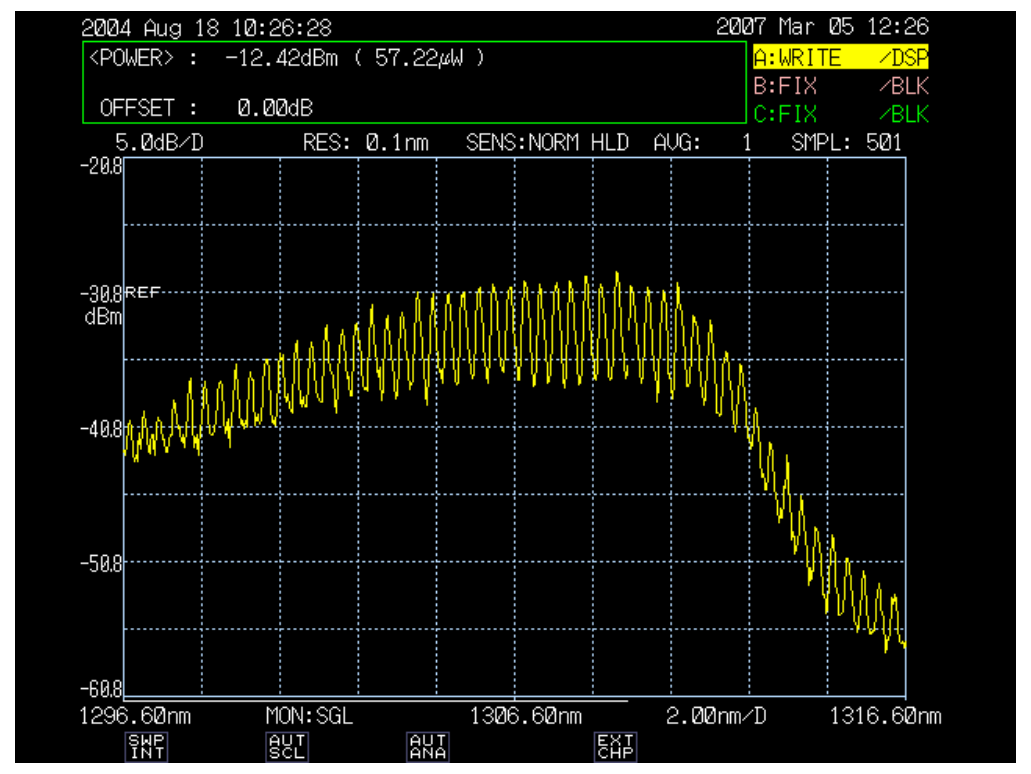

Figure 23: OTDR light source spectrum

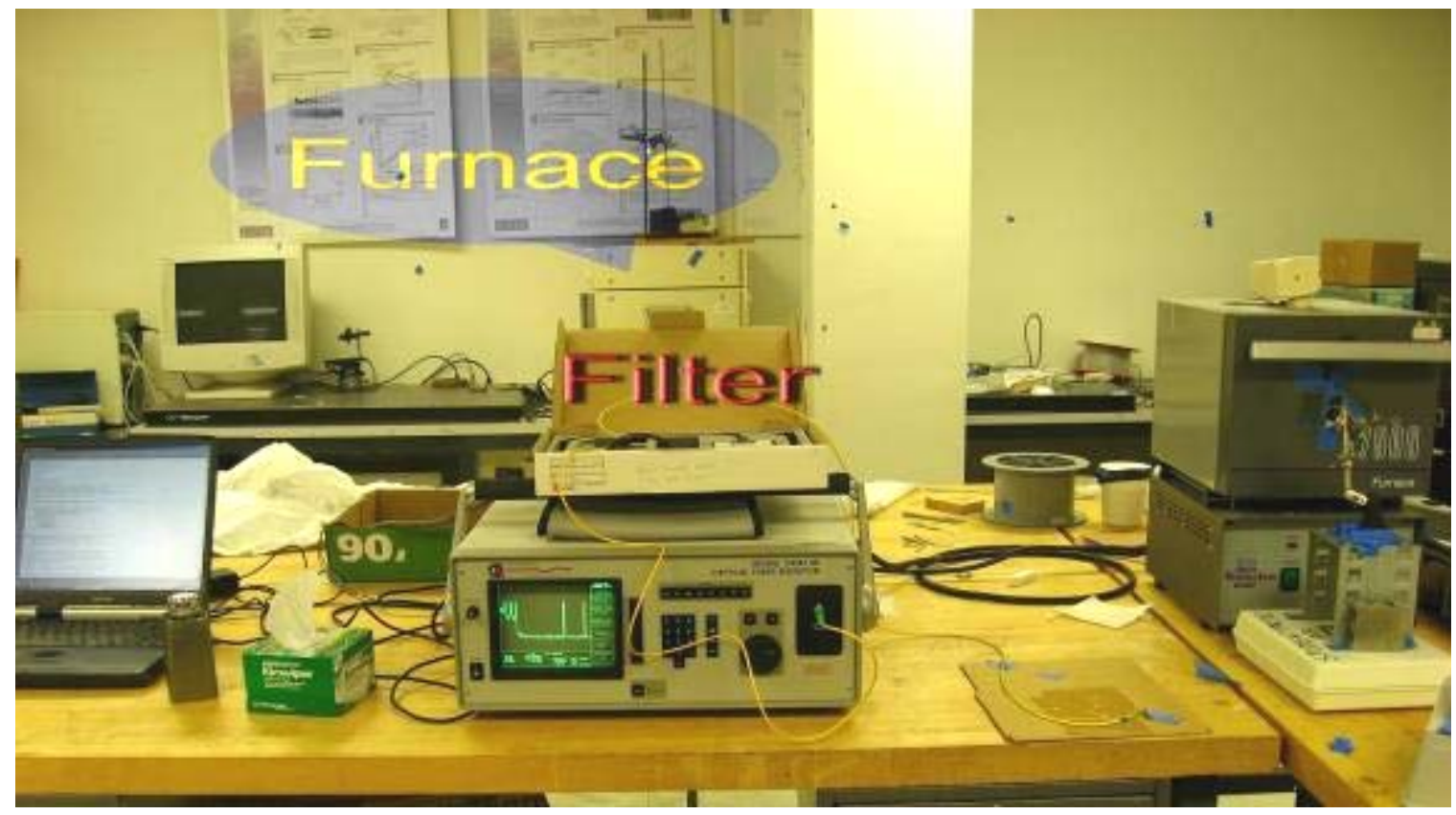

Figure 24: System setup for $1000^{\circ} \mathrm{C}$ testing

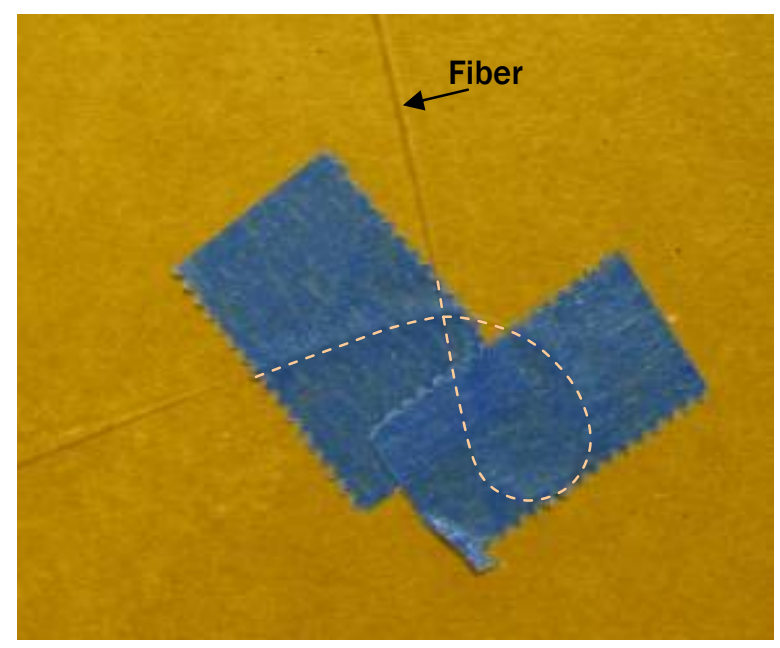

Figure 25: Fiber bending further reduces residual black body radiation 


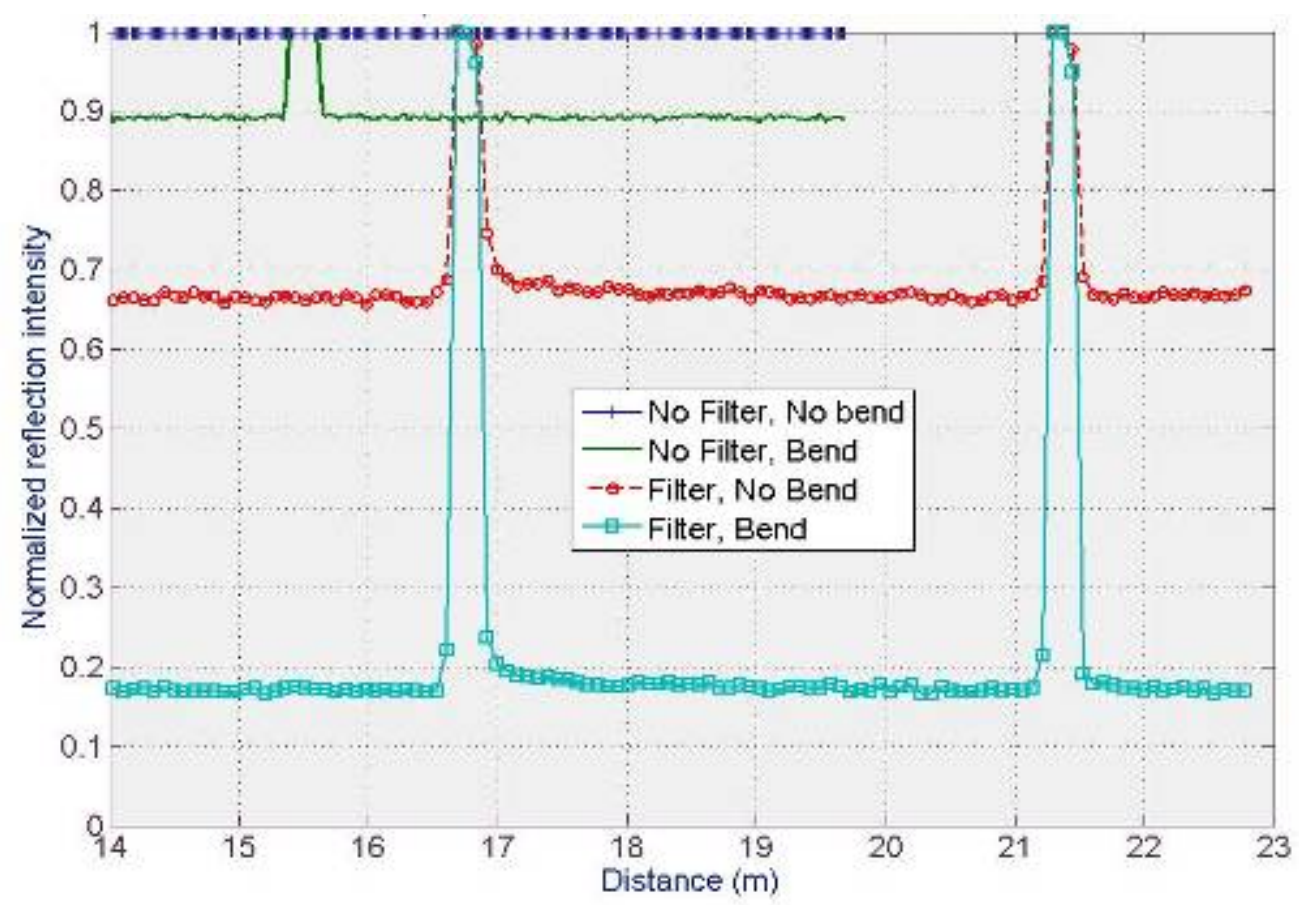

Figure 26: OTDR results under different conditions

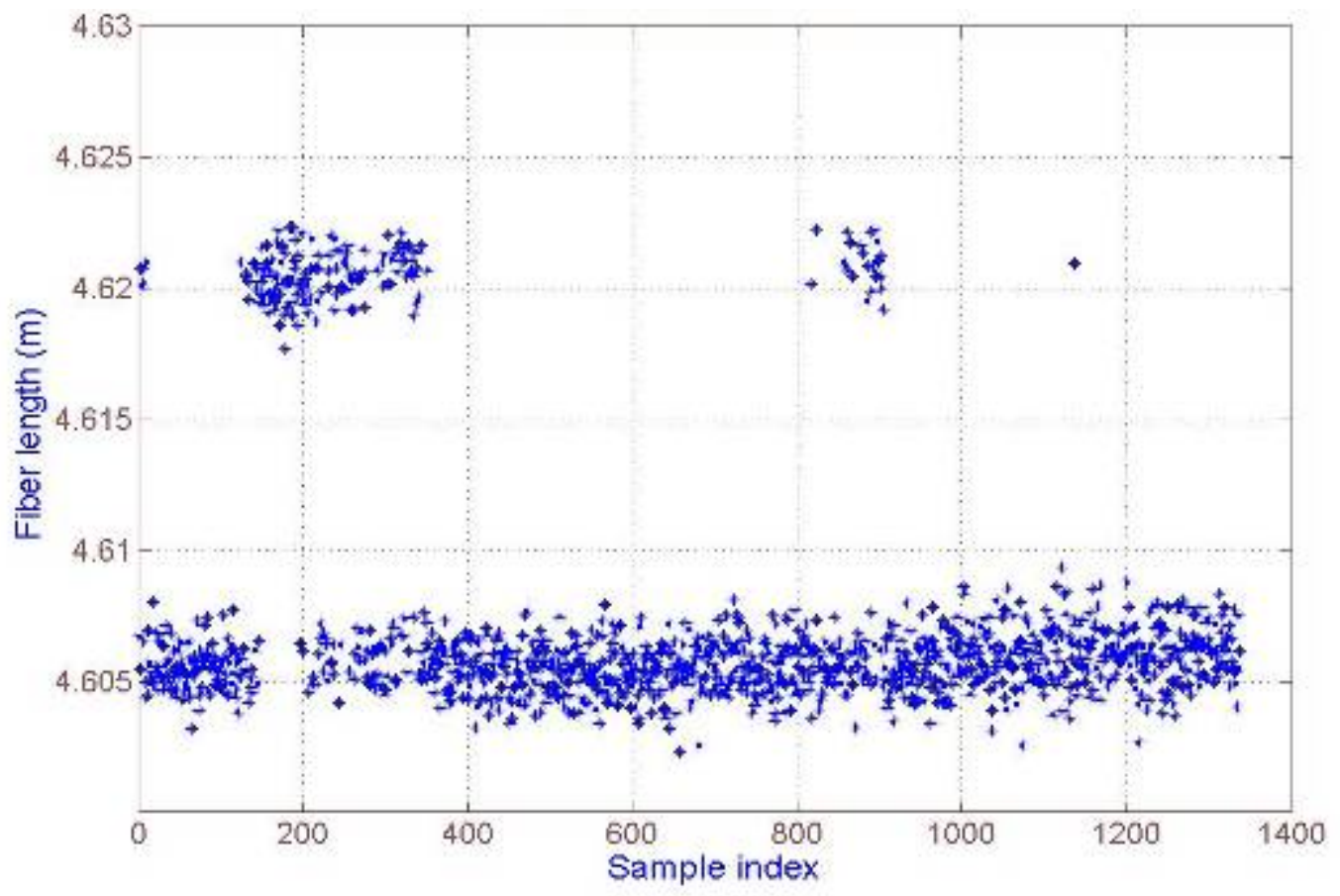

Figure 27: Thickness samples acquired in 24 hour test 


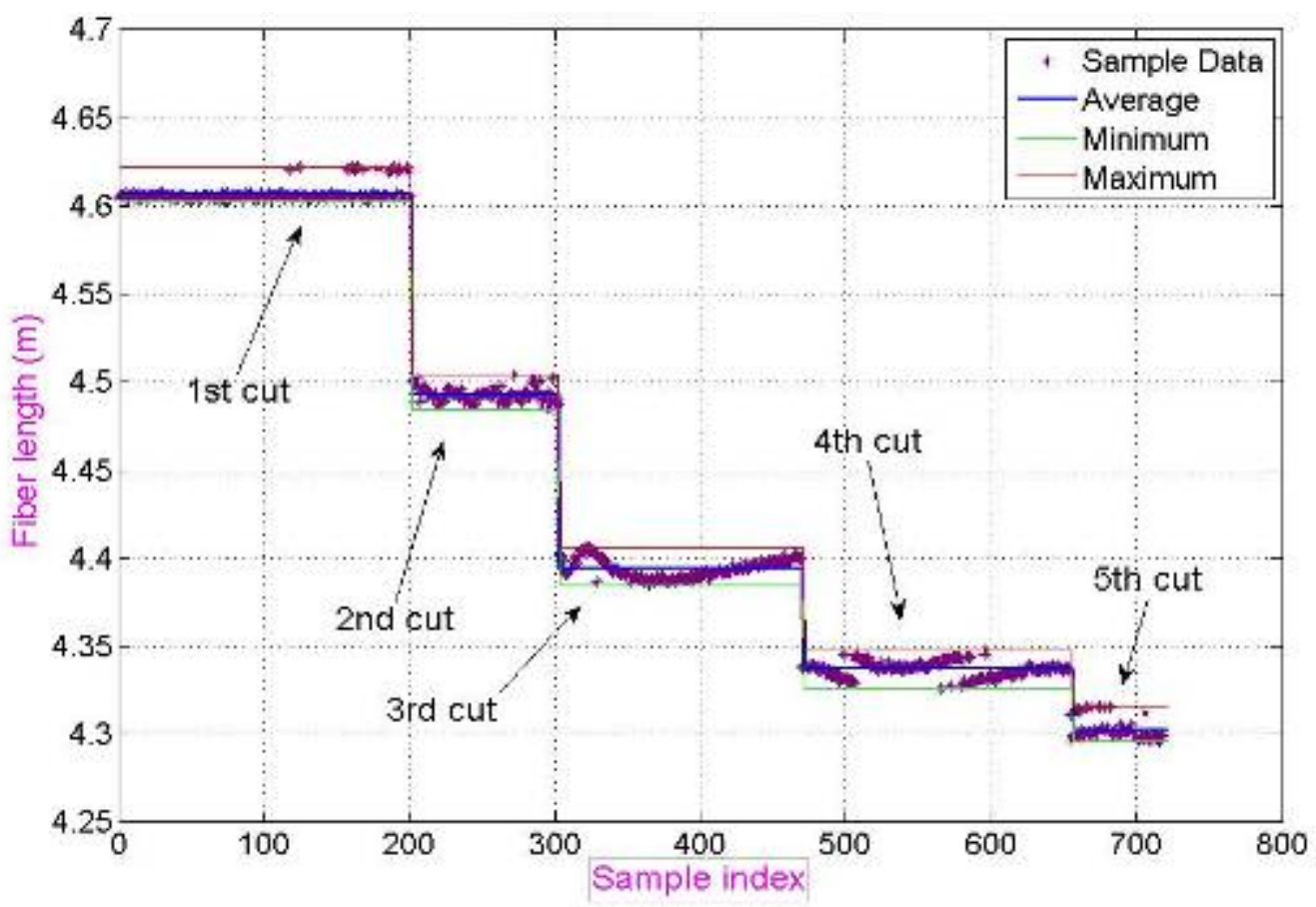

Figure 28: Repeated fiber cut results (non cleaved end)

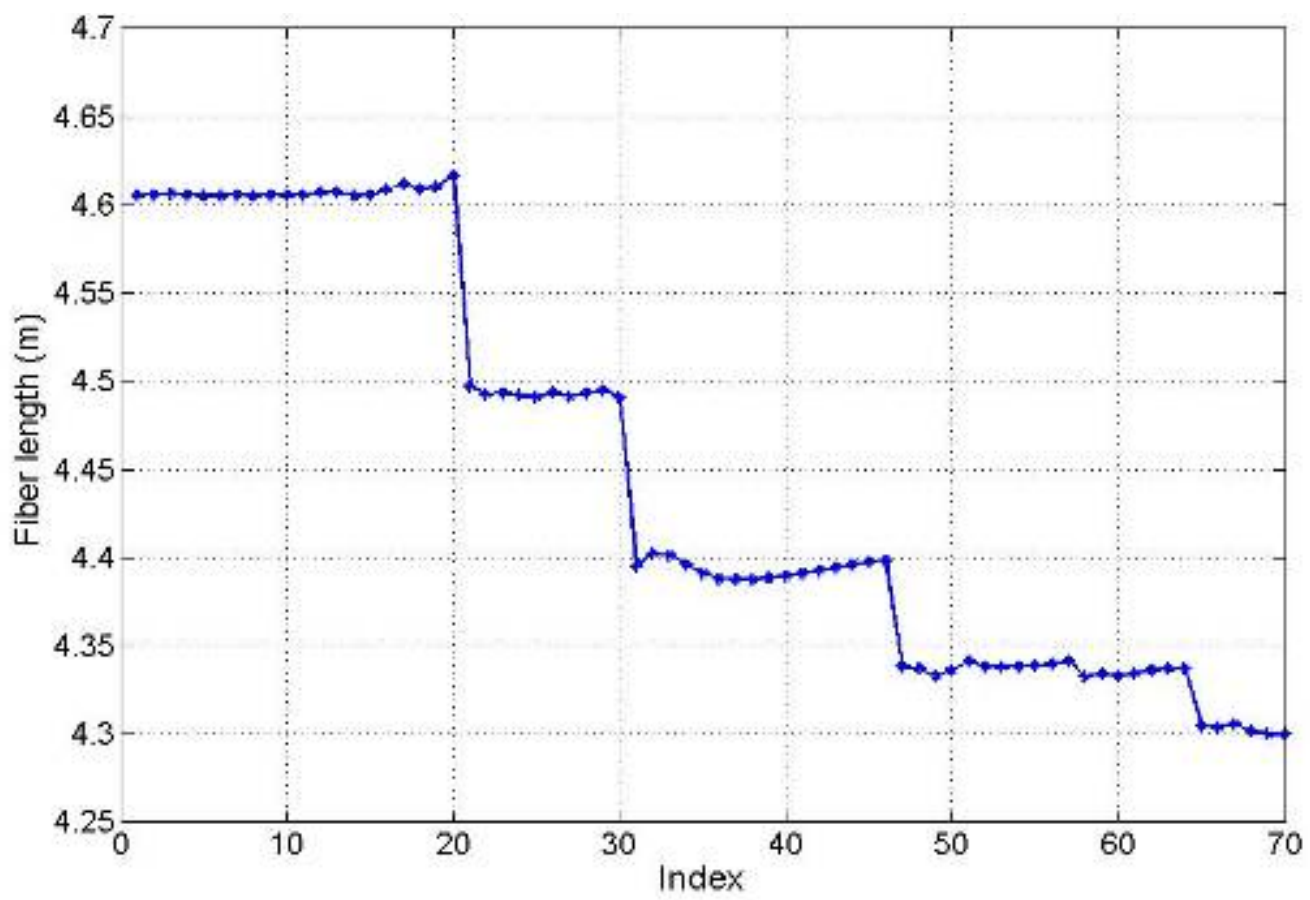

Figure 29: Repeated fiber cut results (non cleaved end) with 10-sample averaging 


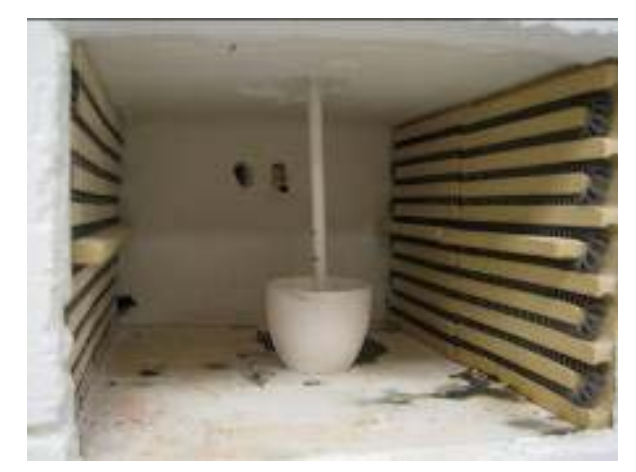

Figure 30: Experimental setup for corrosion experiment. The alumina tube containing the fiber sensor is immersed in sodium carbonate in the crucible

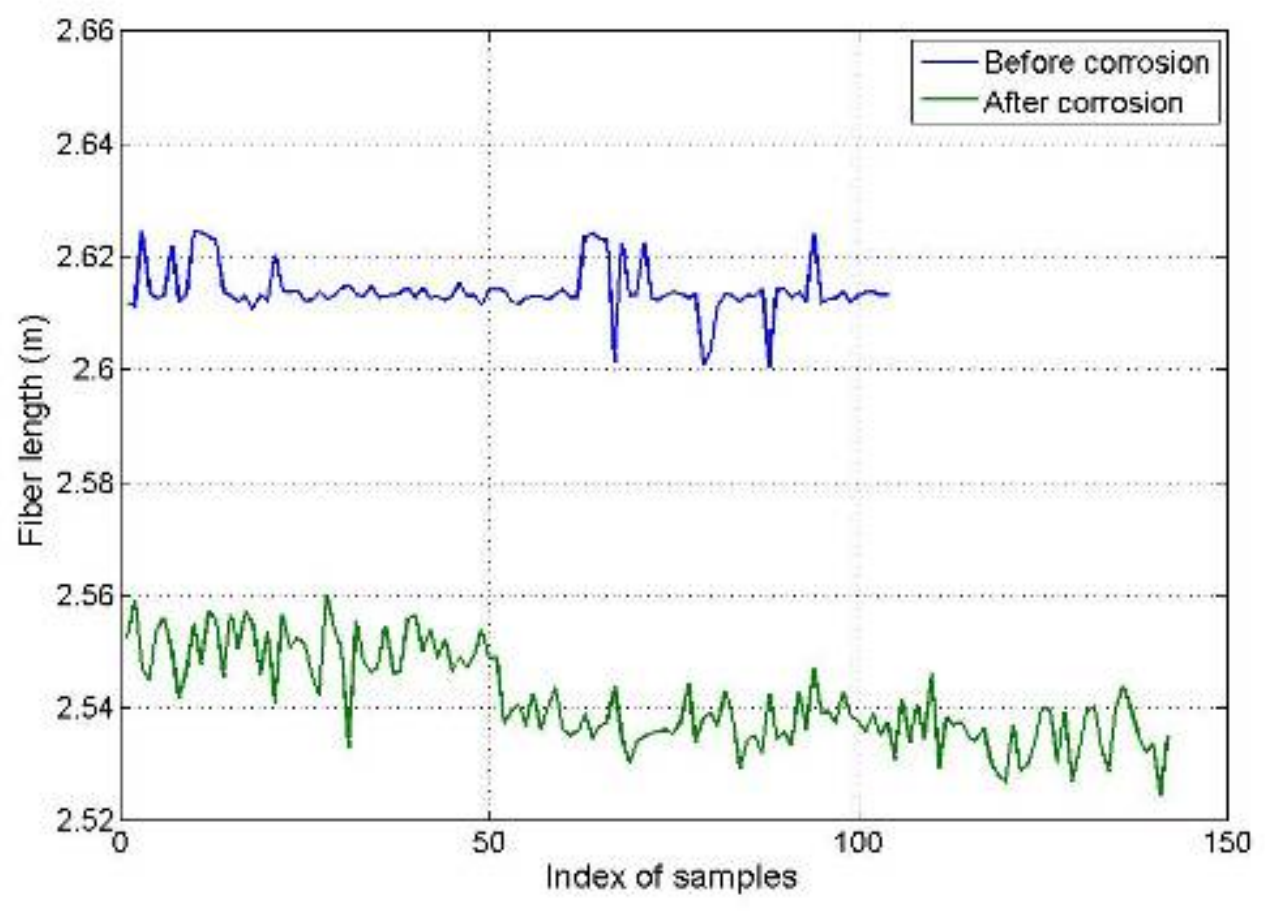

Figure 31: Fiber length measured before and after corrosion

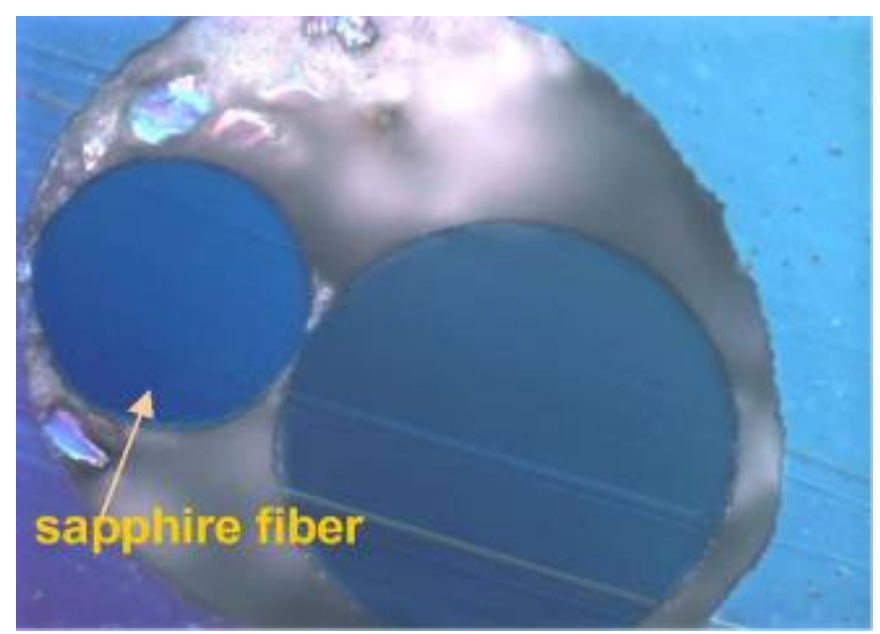

Figure 32: Sapphire end polished by 0.5 micron diamond paper 


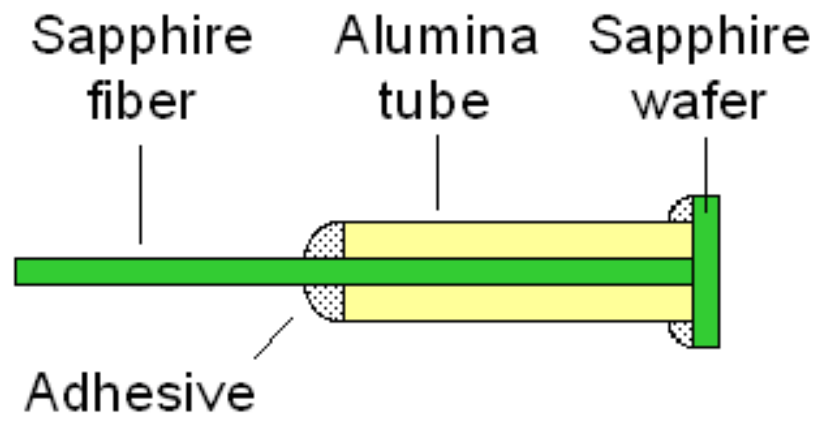

Figure 33: Sensor head structure

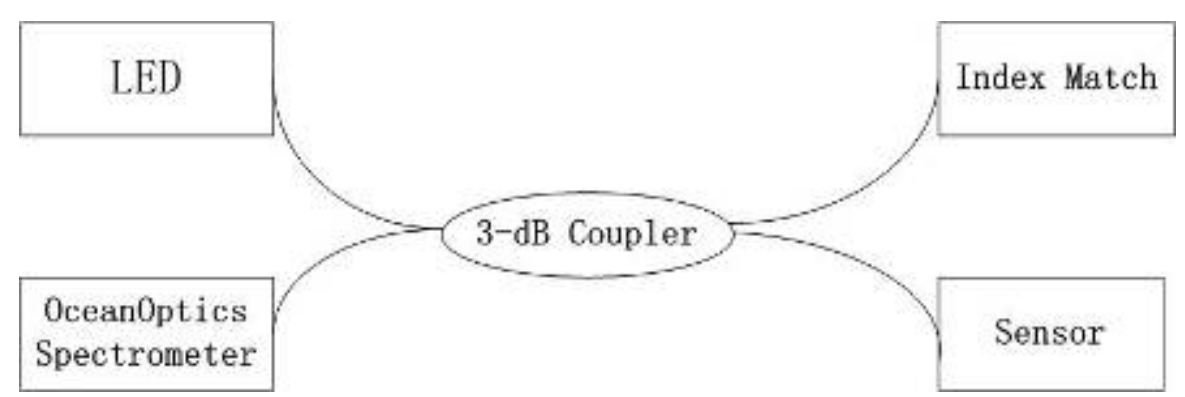

Figure 34: Optical system configuration

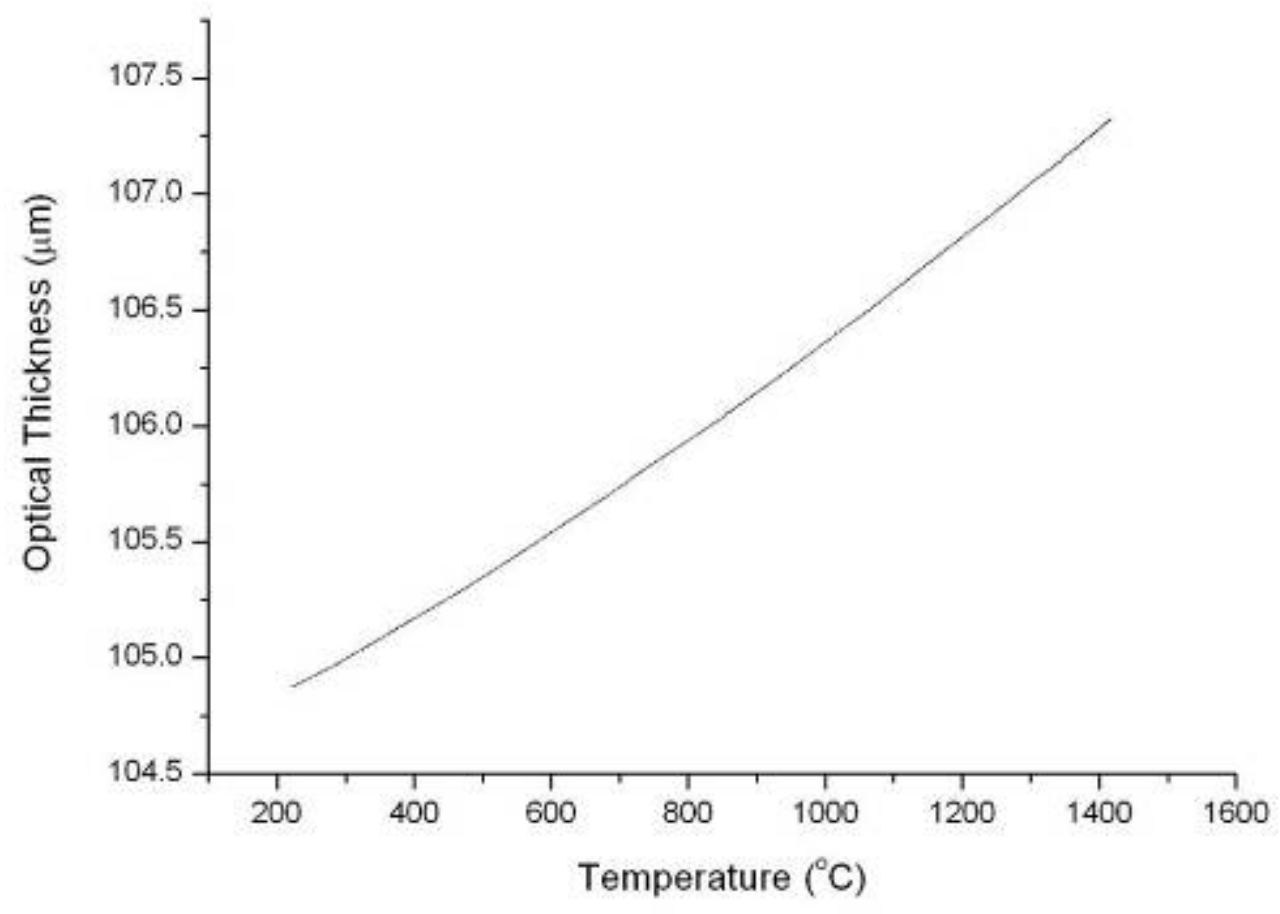

Figure 35: Calibration curve. Although this program leverages technology developed under the DOE program "On-Line Self-Calibrating Single Crystal Sapphire Optical Sensor Instrumentation for Accurate and Reliable Coal Gasifier Temperature Measurement” (DE-FC26-99FT40685), the data presented here is representative of sensors fabricated specifically for the laboratory testing required by this program. 


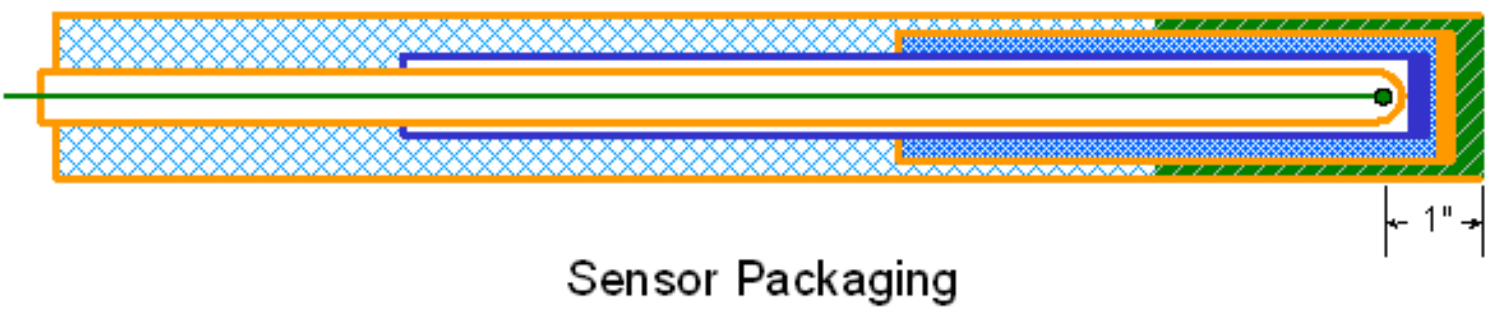

\begin{tabular}{|c|c|c|}
\hline Alumina Tube & Sensor & $8 \times$ \\
\hline Sapphire Tube & $\begin{array}{l}\text { Refractor } \\
\text { Material }\end{array}$ & \\
\hline
\end{tabular}

Figure 36: Packaging scheme. This scheme was designed to support the second field test at TECO (funded by DE-FC26-99FT40685) and due to the improvements from previous designs, will also be used here.

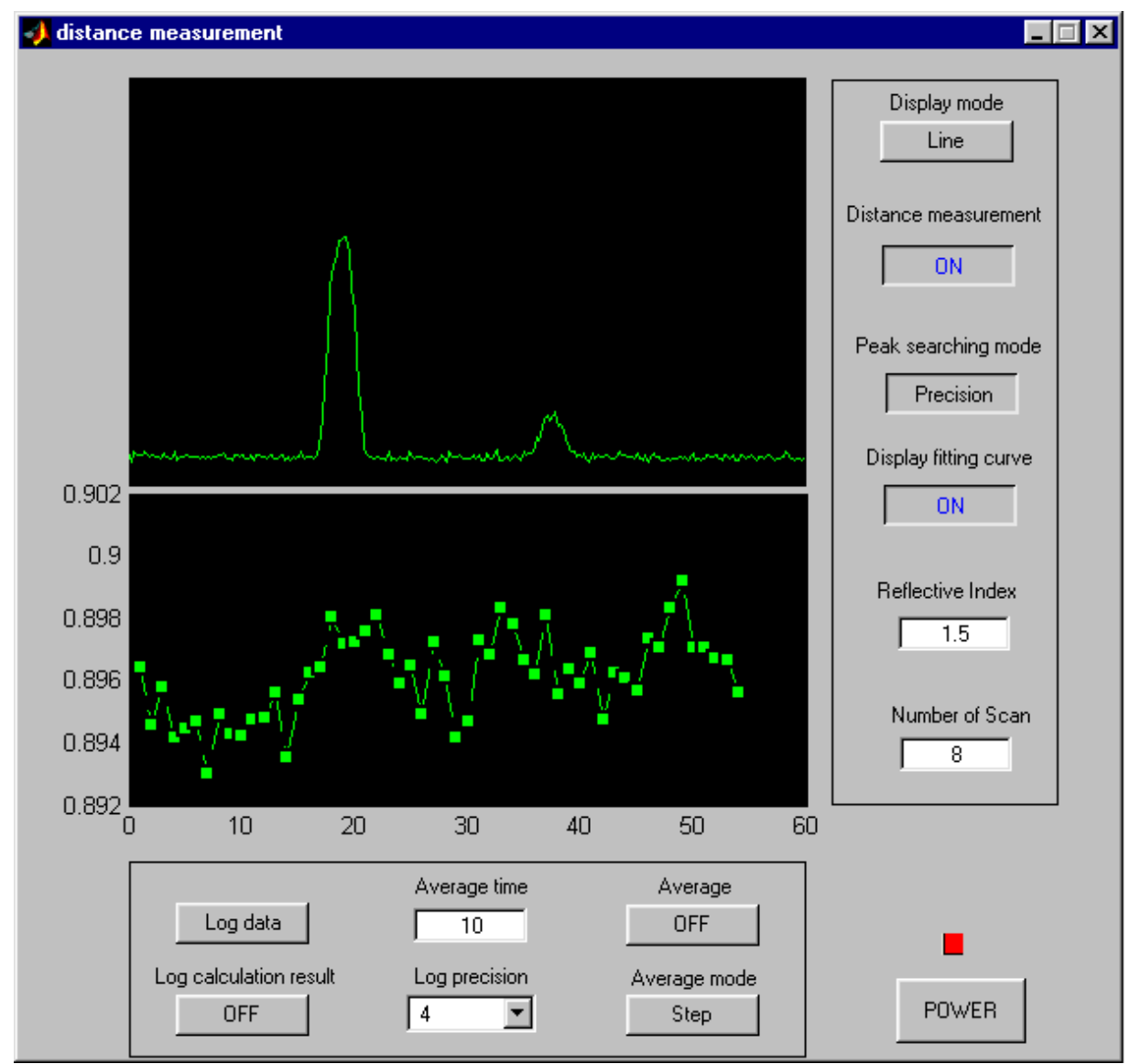

Figure 37: GUI for stand-alone thickness measurement 


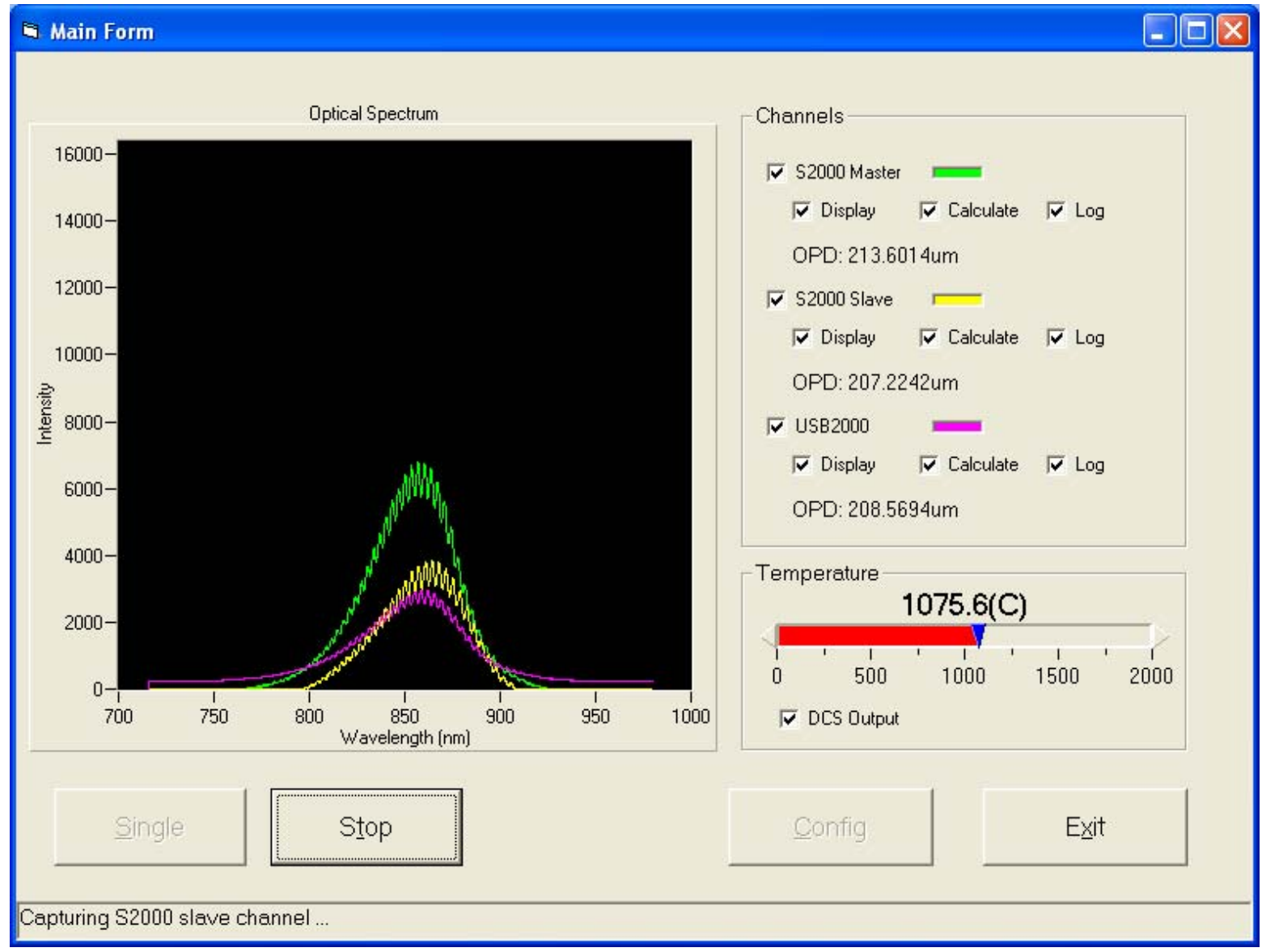

Figure 38: GUI for stand-alone temperature measurement 


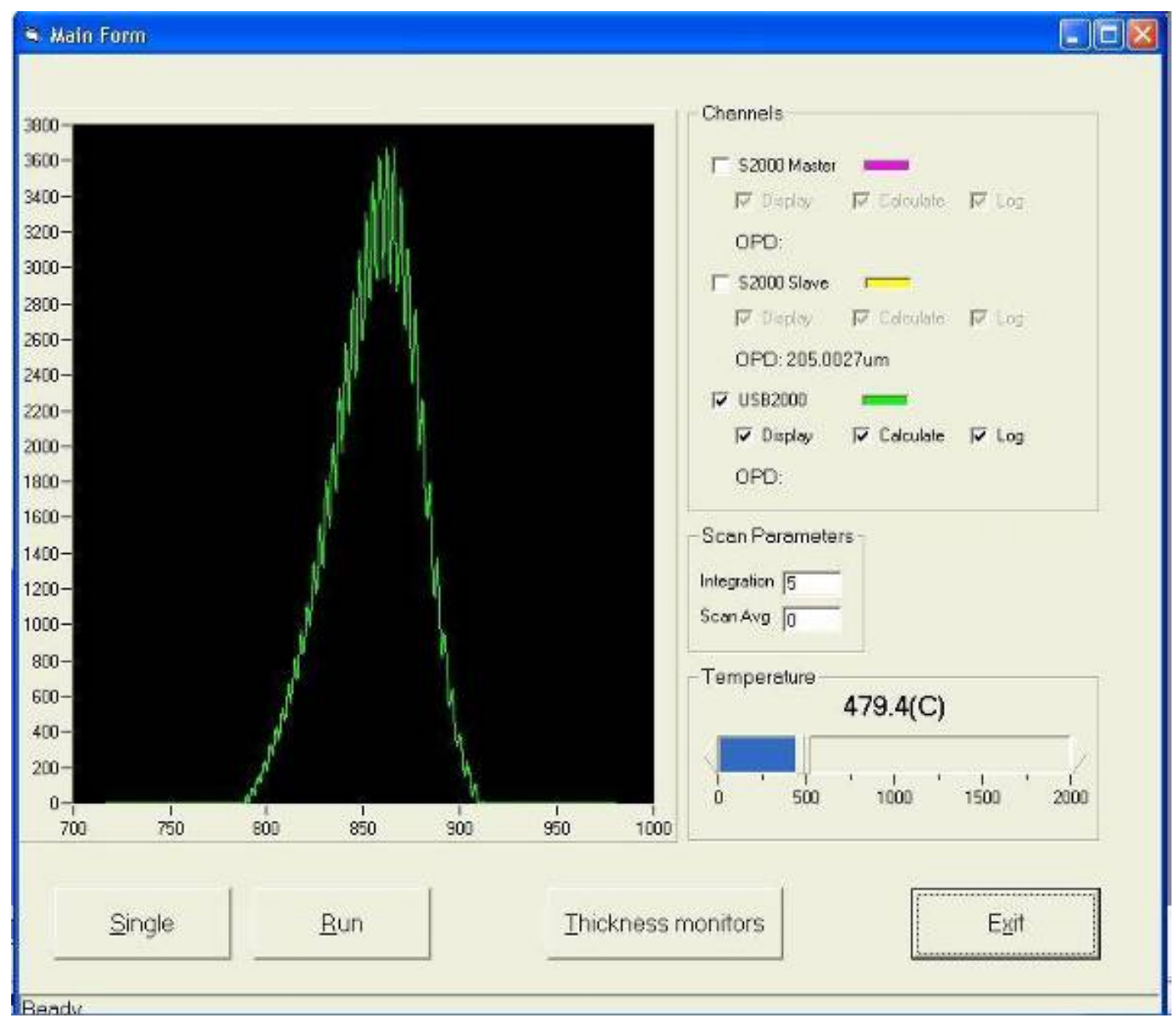

Figure 39: Integrated GUI 


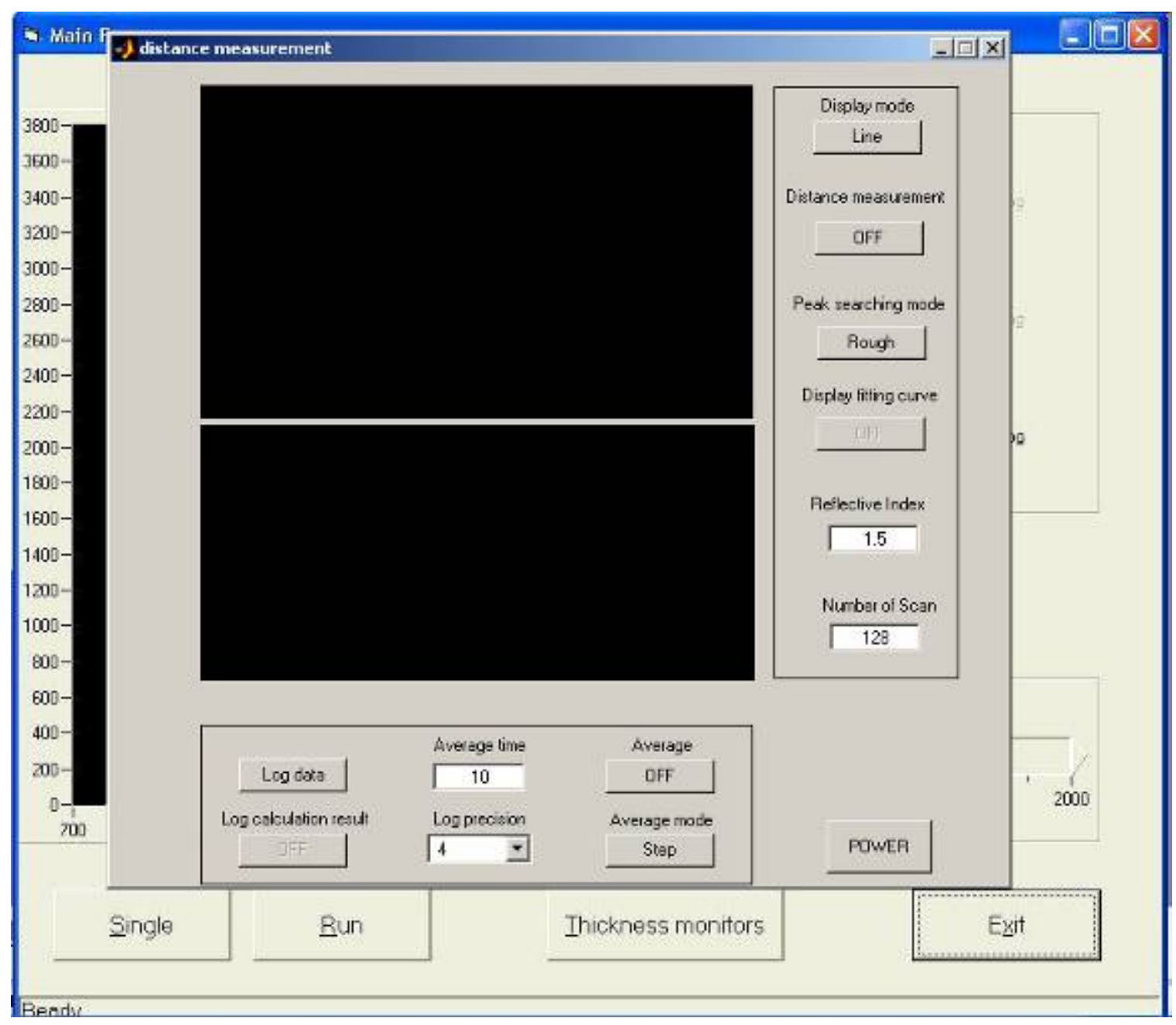

Figure 40: Call of thickness monitoring window 


\section{References}

[1] J. B. Bennett and K.-S. Kwong, "Refractory Liner Materials Used in Slagging Gasifiers," Refractory Applications and News, 9 (5), 20-25, 2004.

[2] S. J. Clayton, G. J. Stiegel, and J. G. Wimer, "Gasification Technologies, Gasification Markets and Technologies - Present and Future: An Industry Perspective," U.S. DOE Report DOE/FE-0447, 2002.

[3] Y. Zhu and A. Wang, "Surface-mount sapphire interferometric temperature sensor," Appl. Opt., 45 (24), 6071-6076, 2006.

[4] F. Shen and A. Wang, "Frequency-estimation-based signal-processing algorithm for whitelight optical fiber Fabry-Perot interferometers," Appl. Opt., 44 (25), 5206-5214, 2005. 


\section{List of Acronyms and Abbreviations}

$\mathrm{A} / \mathrm{D}$, analog to digital

APP, Advanced Pressure Products, Inc.

BPDI, broadband polarimetric differential interferometry

CCD, charge couple device

CPT, Center for Photonics Technology

CTE, coefficient of thermal expansion

EFPI, extrinsic Fabry-Perot interferometer

EMI, electromagnetic interference

FP, Fabry-Perot

FWHM, full width half maximum

GPM, gallons per minute

GRIN, graded index

GUI, graphical user interface

ID, inner diameter

LED, light emitting diode

MM, multimode

MMF, multimode fiber

OD, outer diameter

OPD, optical path difference

OT, optical thickness

OTDR, optical time-domain reflectometer

PC, personal computer

PZT, lead zirconium titanate

RFLWN, raised face long weld neck

SCIIB, self-calibrated interferometric/intensity-based

SLED, super-luminescent light emitting diode

SMF, single mode fiber

SNR, signal to noise ratio

TECO, Tampa electric company

VTPL, Virginia Tech Photonics Laboratory (now Center for Photonics Technology, CPT) 